\title{
The Hadronic Final State at HERA
}

\section{Rajner Mankel DESY}

for the H1 \& ZEUS collaborations 


\section{HERA}

- HERA ep collider at DESY: a unique machine

- Presently the only operating high energy collider in Europe

- HERA collides protons and electrons/positrons at $\sqrt{s}=318 \mathrm{GeV}$

- HERA-II run features upgraded luminosity and polarization

- Colliding beam experiments: $\mathrm{H} 1$ and ZEUS

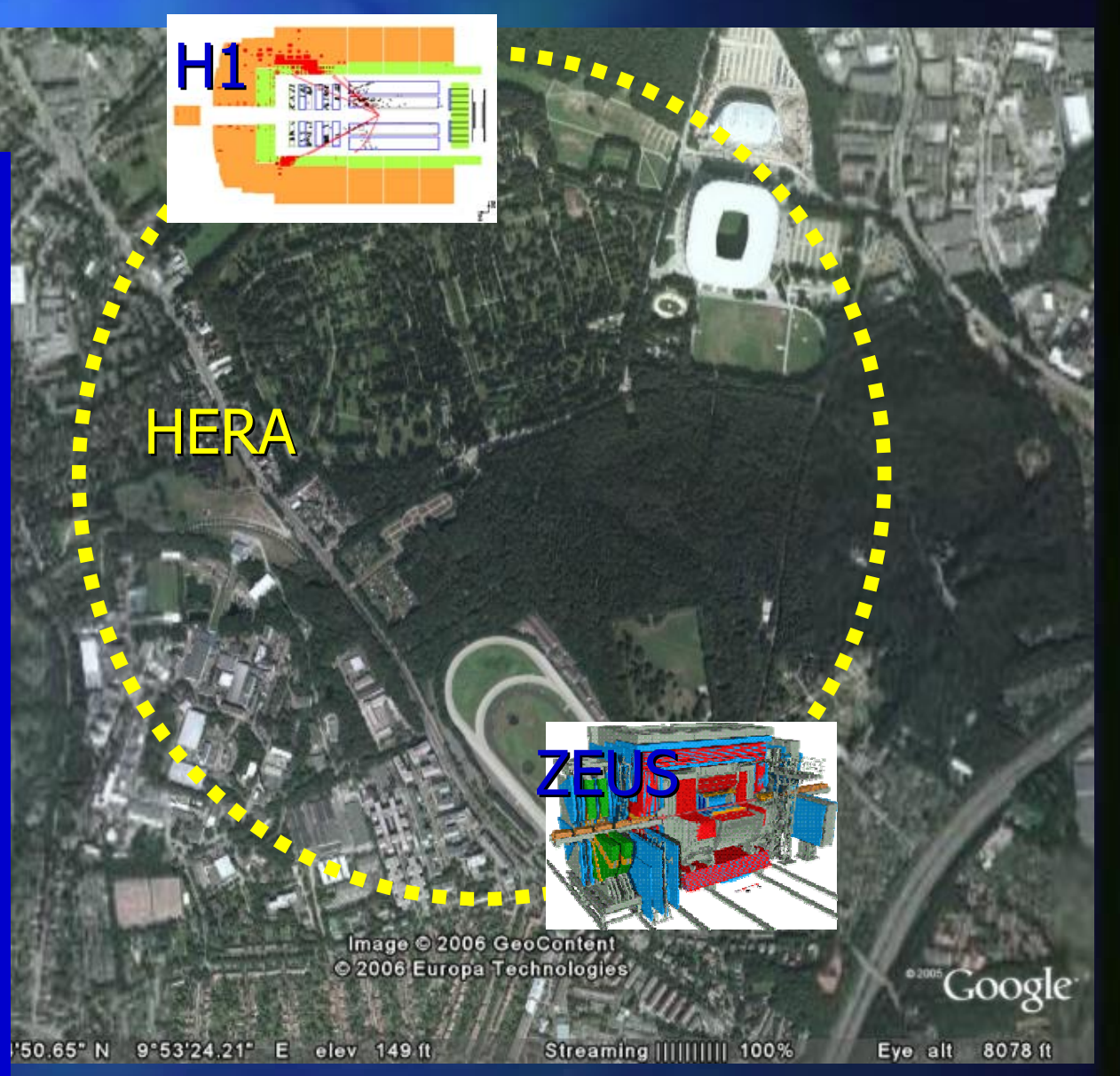




\section{HERA LUMMjijosity}

\lrcorner$\quad H E R A-I I$ has been surpassing all previous Juminosity achievenents

$\checkmark$ Already the HERA-II e-p run (Dec 04-Jun 06) has delivered more colljsions thals six years of HERA-I (1995-2000)

- Since end Jun 06, machine has switched back to $e^{+} p\left(\sim 40 p^{-1}\right.$ since)

- $\mathrm{O}\left(500 \mathrm{pb}^{-1}\right)$ per expt expected by end of data-taking in mid 2007

- Also analysis of HERA-I data is still going strong

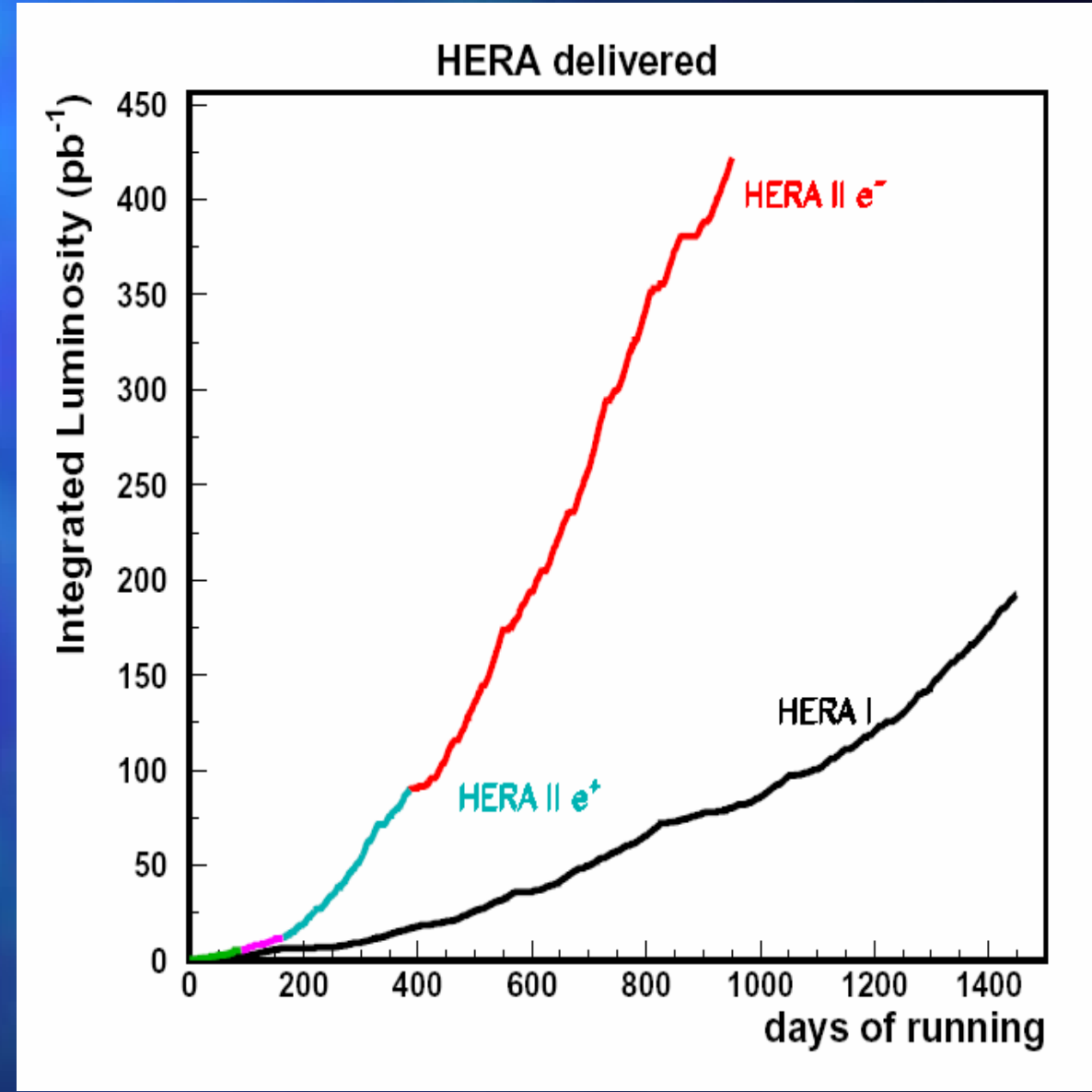




\section{Typical Structure of Hadronic Final States at HERA}

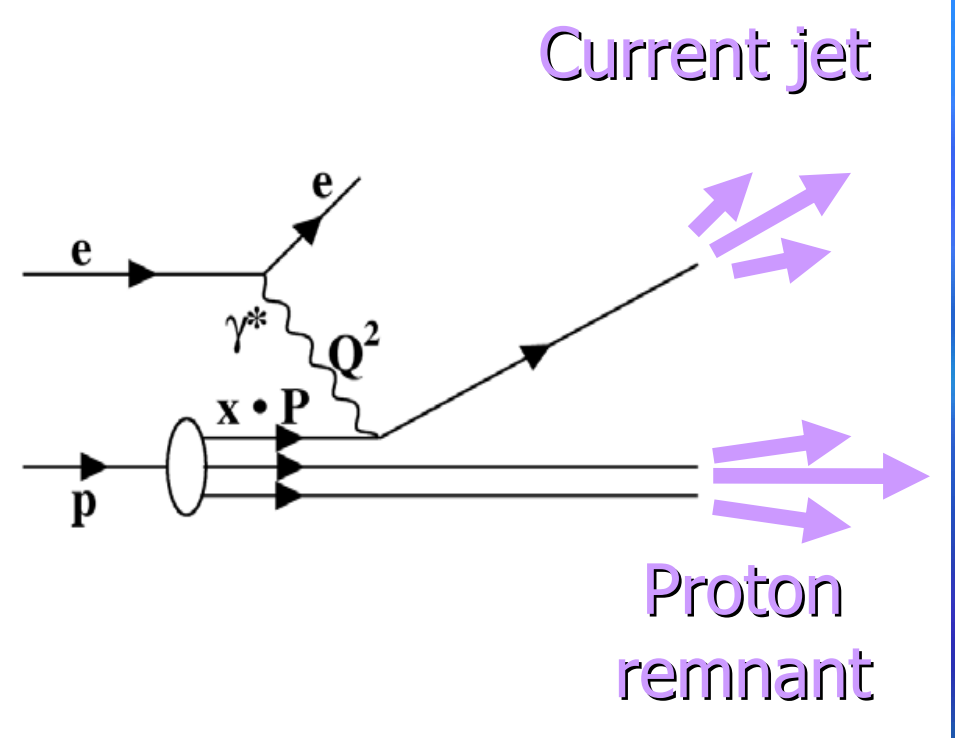

$\sqcup \mathrm{Q}^{2}$ : virtuality of exchanged photon (boson)

- $\mathrm{Q}^{2}>1 \mathrm{GeV}^{2}$ : deepinelastic scattering (DIS)

- $\mathrm{Q}^{2}<1 \mathrm{GeV}^{2}$ : photoproduction (PHP)

- $x\left(x_{B j}\right)$ : fraction of proton momentum carried by struck quark 


\section{Comparison of Hadronic Final State}

\section{Structure}

hadron-hadron interaction

$e^{+} e^{-}$interaction

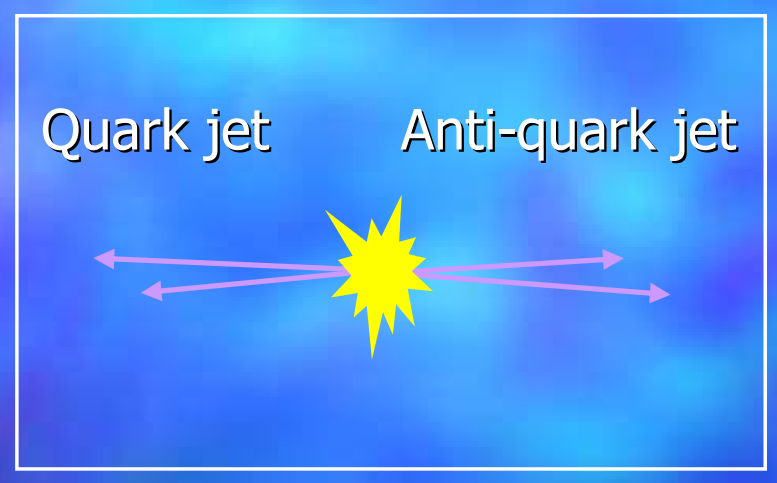

$e^{ \pm p}$ interaction

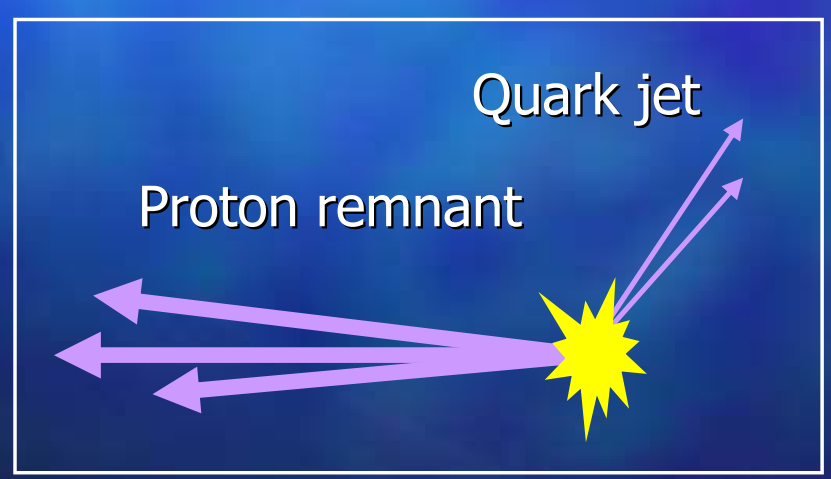

$\Rightarrow$ contains main features of energetic hadron interaction (proton remnant)

$\Rightarrow$ less complex than hadron-hadron interaction

$\Rightarrow$ clean reconstruction of kinematic variables

$\Rightarrow$ ideal laboratory for studying QCD 


\section{Colljdjing Beamn Detectors}

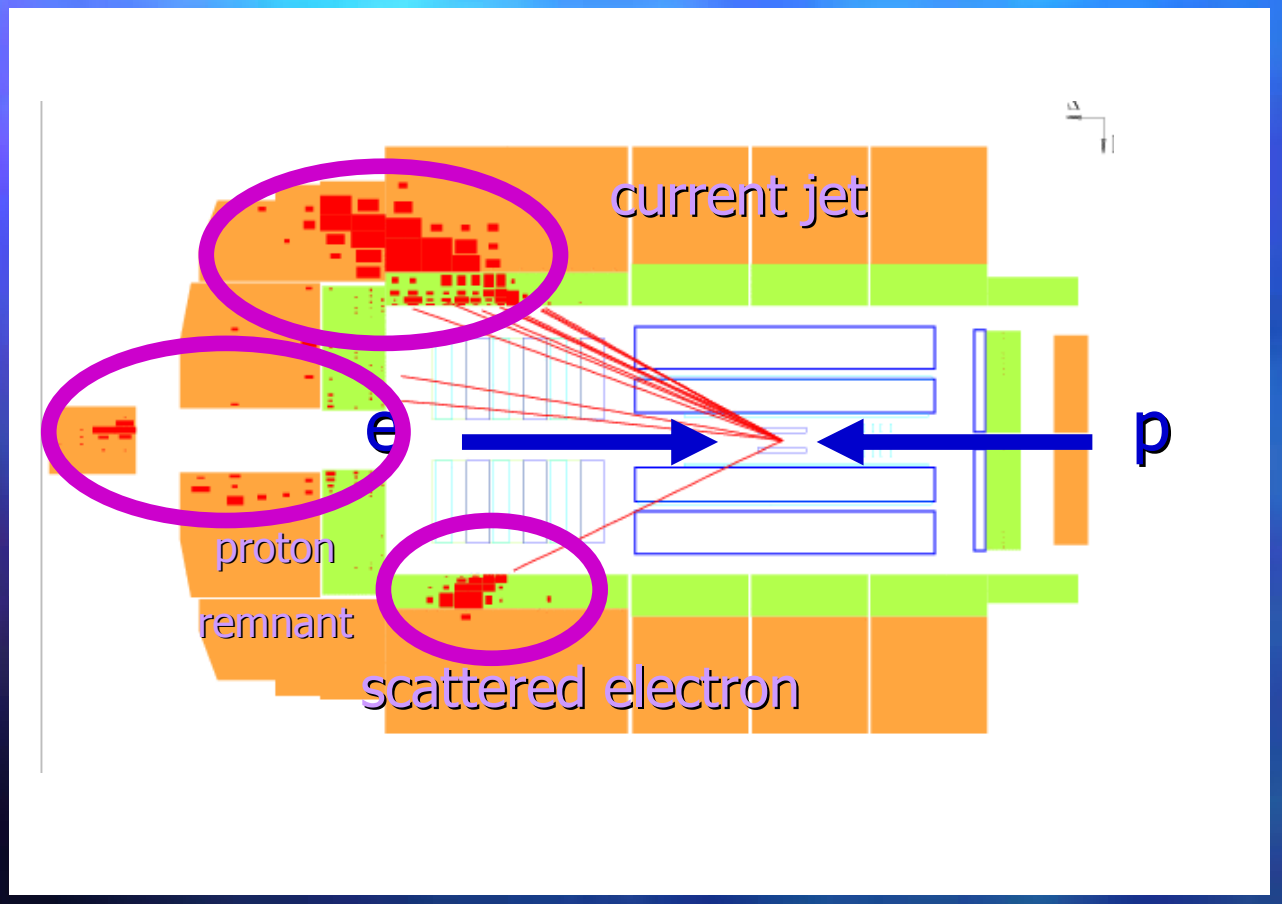

- Colliding mode detectors can generally measure current jet \& scattered electron very well ("central region")

- in these areas, also theoretical approaches are tested \& tuned best

- in PHP, the scattered electron usually escapes along the beam pipe

- The proton remnant emerges close to beam pipe $\&$ is less accessible

- these areas also pose big challenges to theory

- Additional jets can arise from more complex processes 


\section{Some "Frontjer" Questions Related to Hadronjuc Final Stajte}

\lrcorner How relevant are higher orders in perturbative QCD?

\lrcorner How well do we understand the workings of QCD in the forward area?

- Can we distingujsh evolution schemes in parton cascades?

- At which accuracy can we describe production of heavy flavor? 


\section{Outljine}

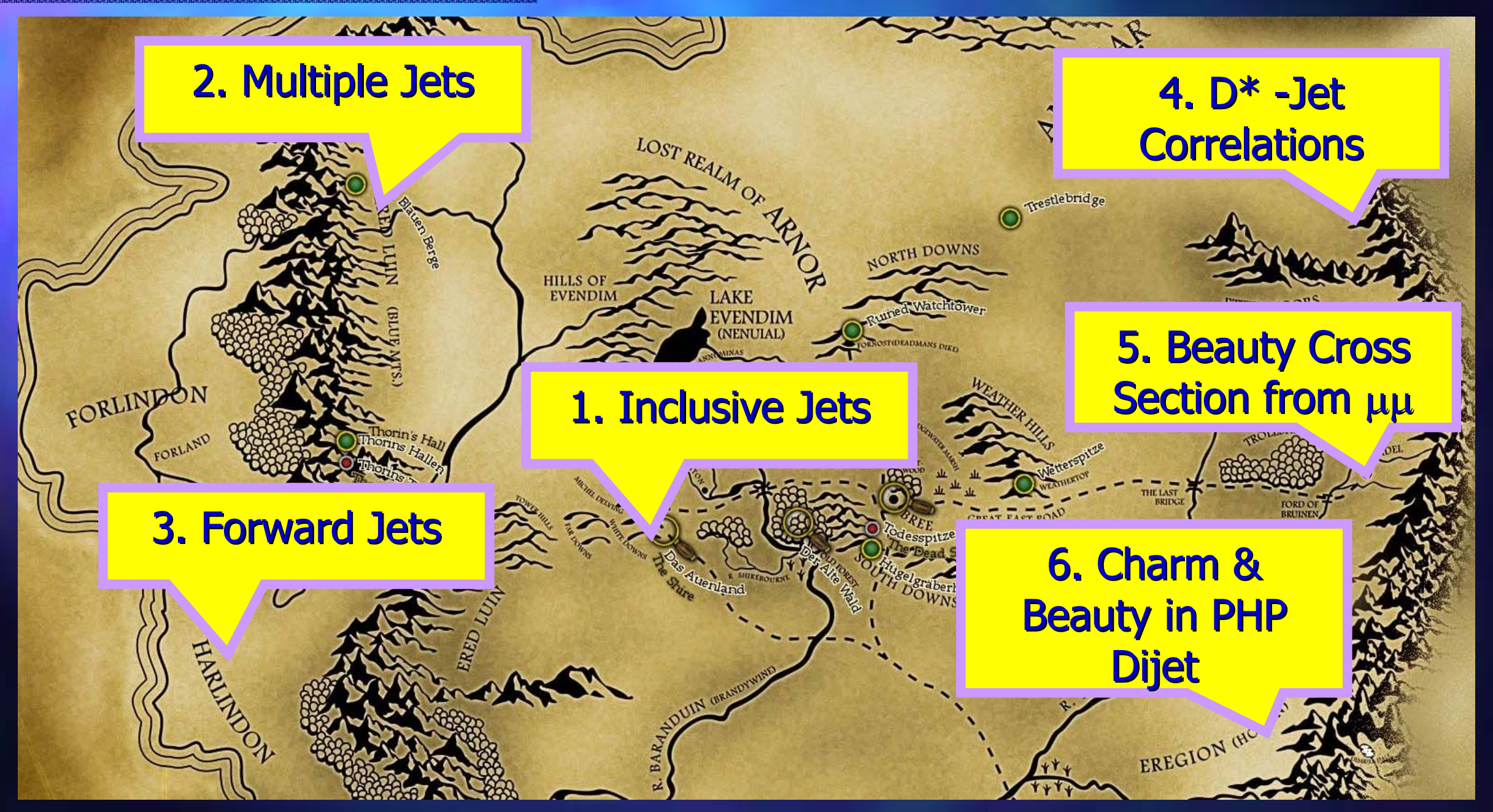


1.
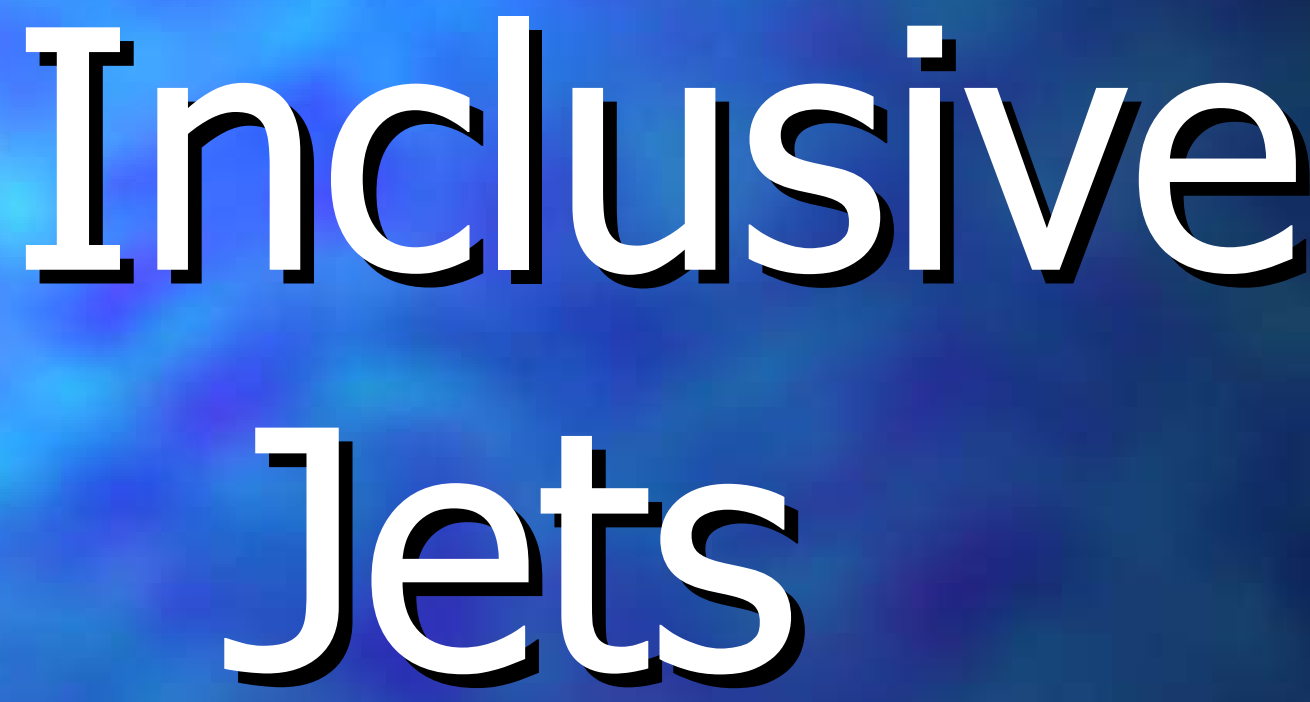


\section{Insclusive Jet Production in NC DIS}

\lrcorner Jet search in Breit firame

\lrcorner virtulal photon purely space-like, defines longitudinal direction

- optimal separation of proton remnant \& recoilling parton

$\square$ High Ejet

$\Rightarrow$ majinly sensitive to hard QCD processes

$\Rightarrow$ ideal testing ground for PQCD

$\Rightarrow$ Experiment and NLO calculations agree over five orders (!) of magnitude in the $\mathrm{Q}^{2}$ spectrum

$\Rightarrow$ Impressive success for QCD theory

$\Rightarrow$ Experimental uncertainty (mainly jet energy scale) tends to be smaller than theoretical uncertainly of NLO calculations

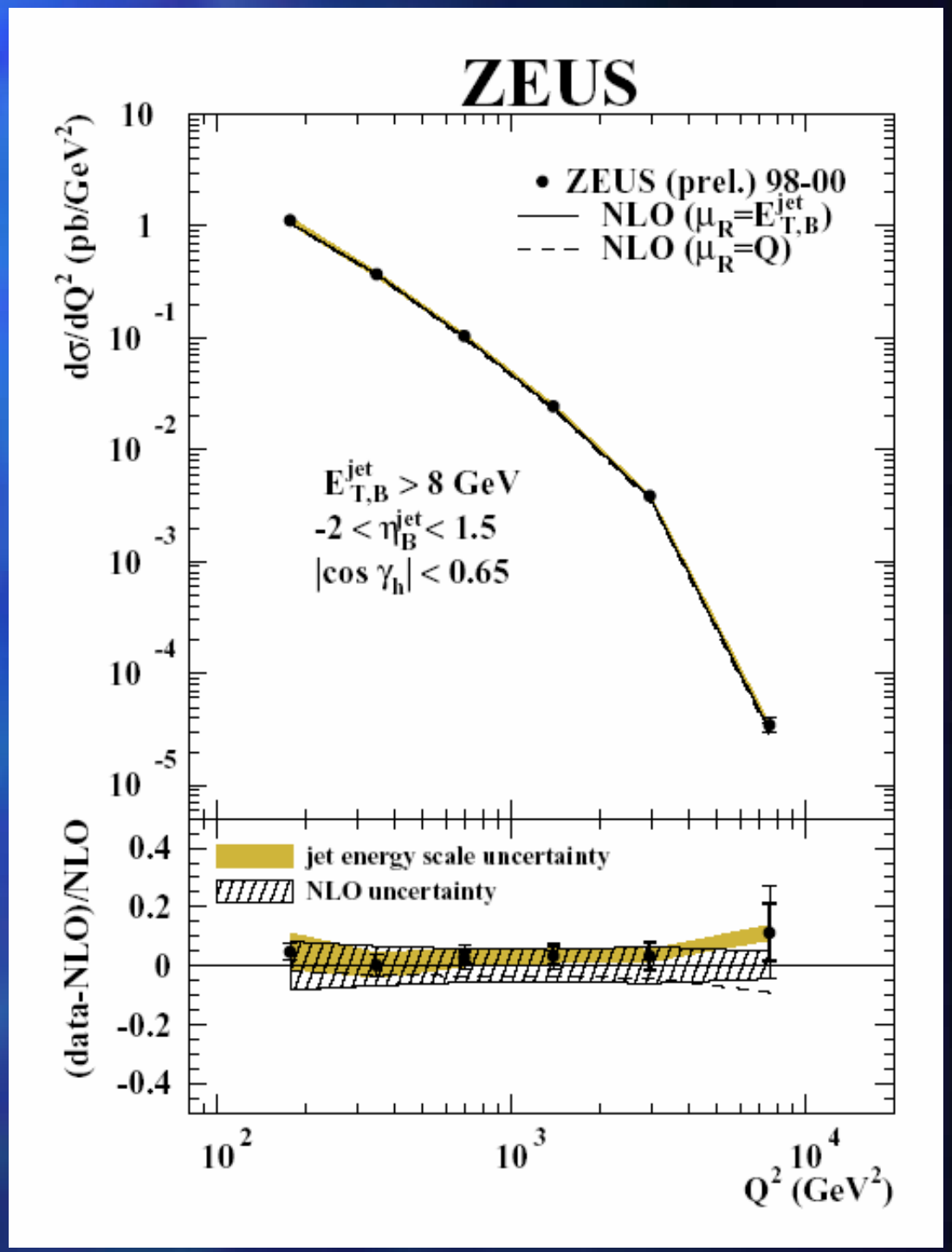




\section{Inclusive Jet Production in NC DIS (contt'd)}

\lrcorner Ejet $_{T, \mathrm{~B}}$ dependence becomes less steep as $Q^{2}$ increases

$\Rightarrow$ Measurrements well described by NLO QCD

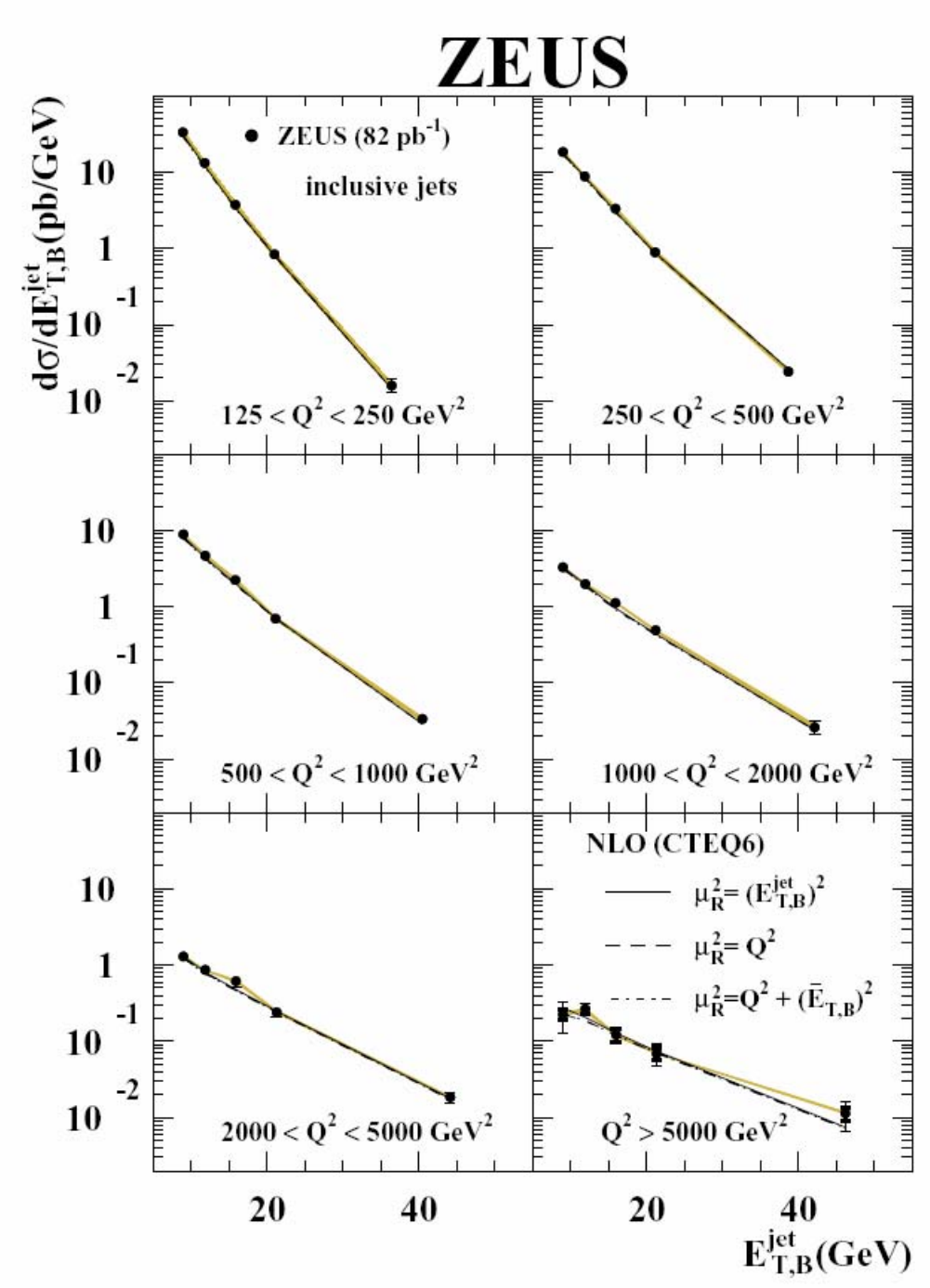




\section{Inclusive Jet Production $\left(\operatorname{con} t^{\prime} d\right)$}

\lrcorner Diffierential cross sections vs $E_{T_{1, B}}$ jet and $Q^{2}$ can be used to extract strong coupling constant

$\Rightarrow$ Running of $o_{s}$ clearly seen

$\Rightarrow$ Shape agrees with theoretical expectation

$\Rightarrow$ Value of $\alpha_{s}\left(M_{Z}\right)$ in accord with world average

$\Rightarrow$ competitive precision

- Measuring whole Q range in one analysis avoids systematic uncertainties that arise when combining different experiments
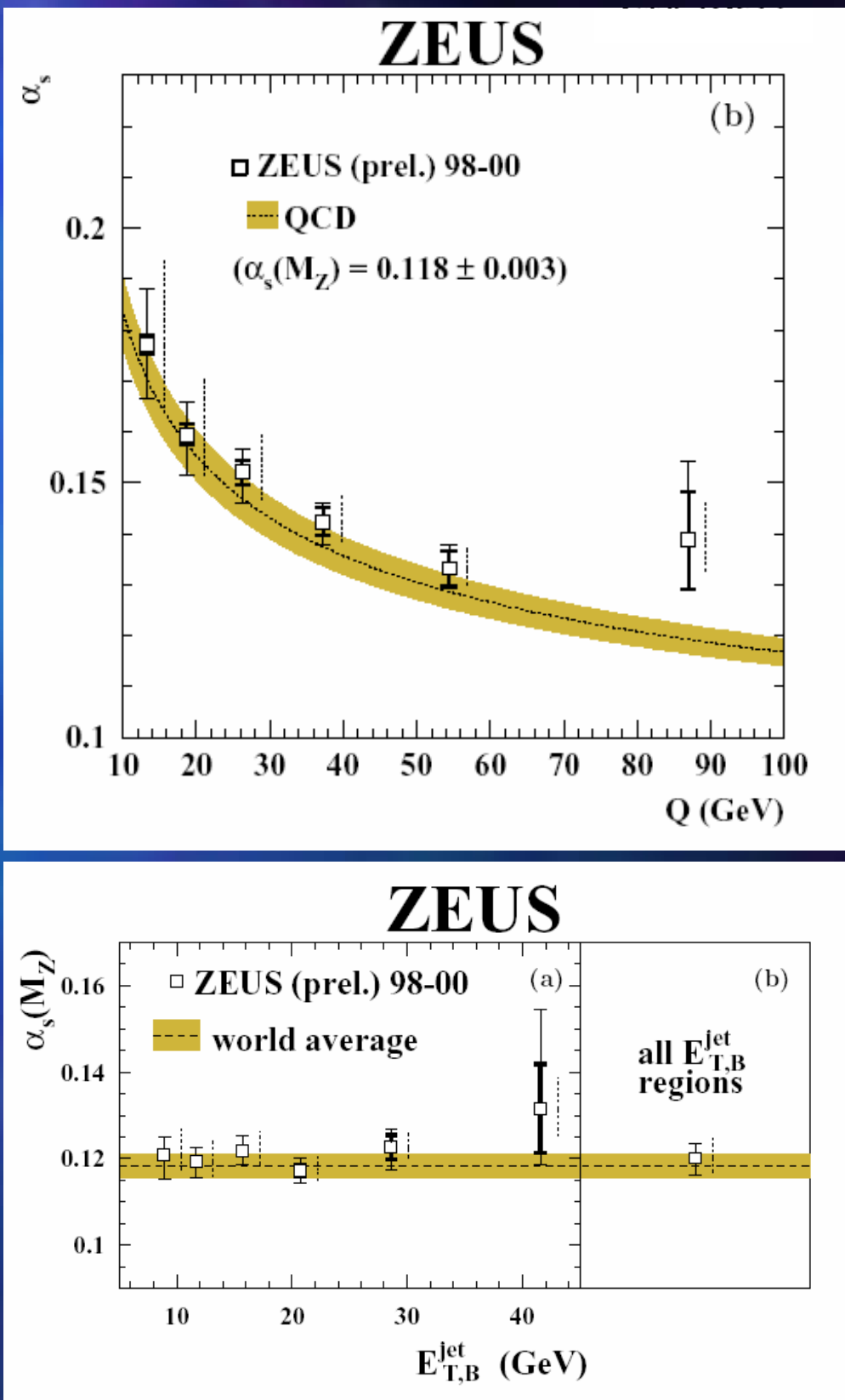

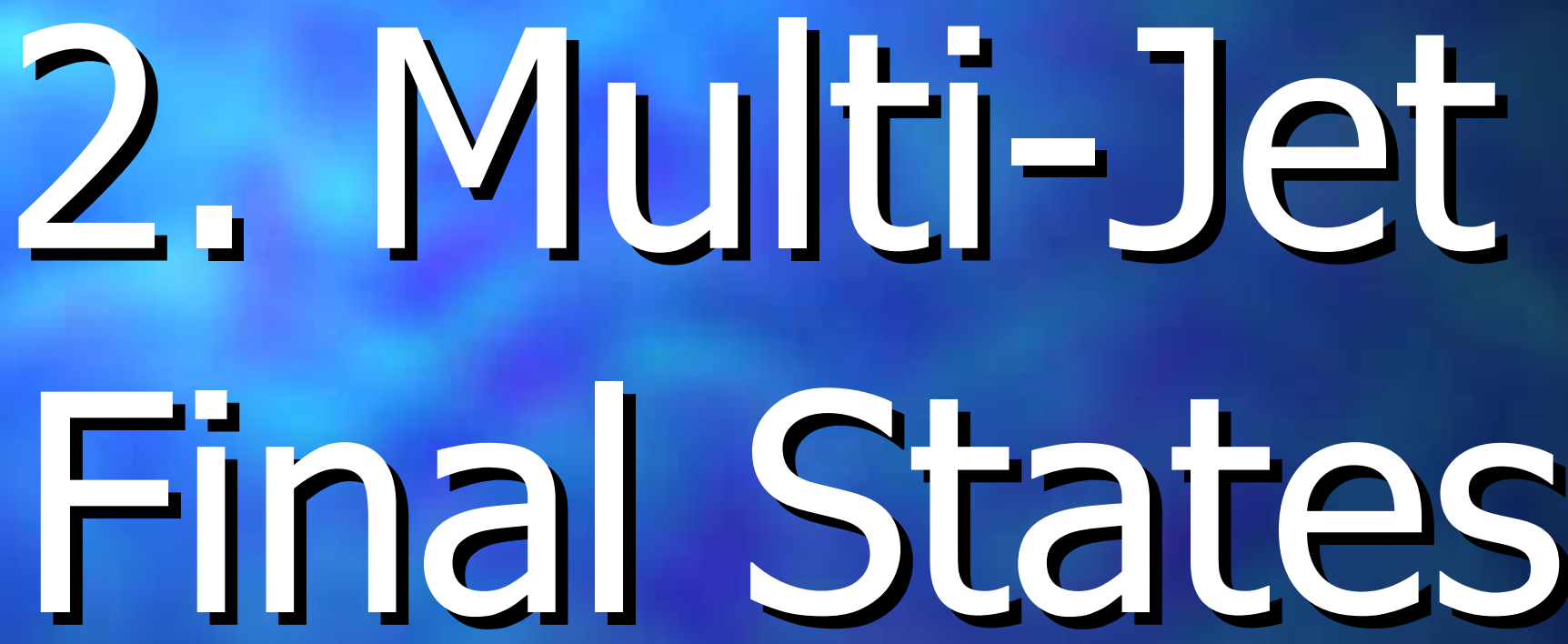


\section{Mulțjet Final States}

\lrcorner Historical note: in 1979 , the first djirect observatjon of the gluon was made at DESY, as a third jet in $e^{+} e^{-}$annihilation

- resulting from hard gluon radiation

- could estimate $\alpha_{s}$ from relative rate

$\downarrow$ Three-jet signatures can be seen as the modern HERA equivalent of this measurement

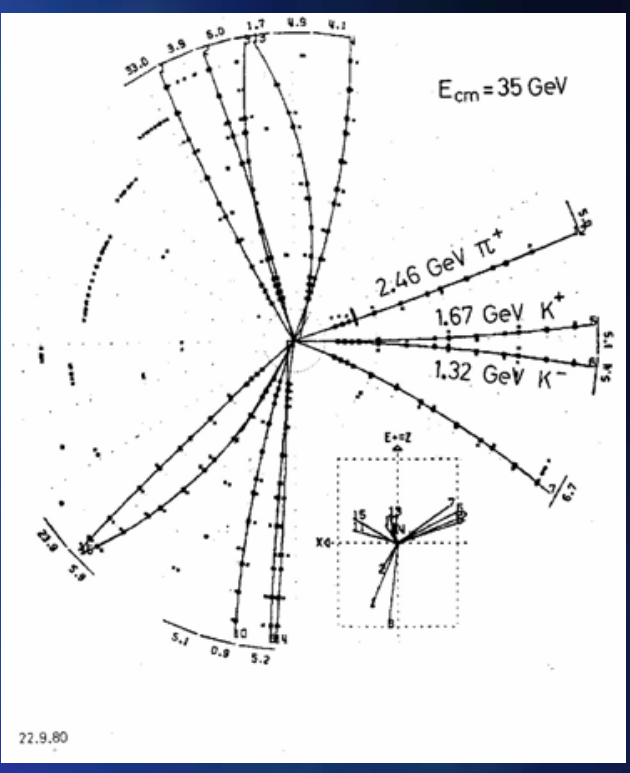

- in Breit frame similar quite picture as in $\mathrm{e}^{+} \mathrm{e}^{-}$

- one jet emerging from hard gluon radiation

- can measure $\alpha_{\mathrm{s}}$ from ratio of 3-jet : 2-jet production

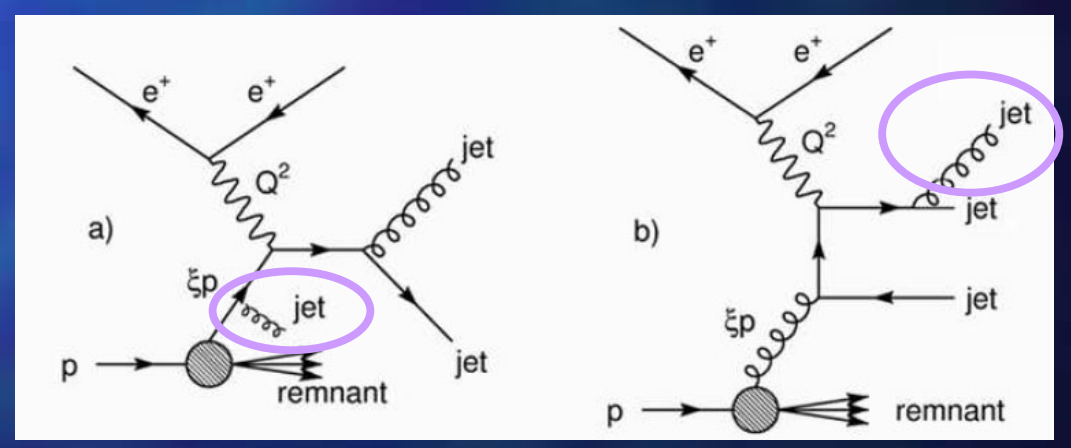




\section{Tri-Jet Final State: Jet Energy Spectra}

\lrcorner Jets classifjed accordling to decreasing transverse energy $\mathrm{E}_{\mathrm{T}, \mathrm{B}} \mathrm{B}^{\mathrm{j} e t}$

$\Rightarrow$ Good description by NLO* in $\mathrm{O}\left(\alpha_{s}{ }^{3}\right)$, even at low $E_{T, B}$ jet

*NLOJET with CTEQ6

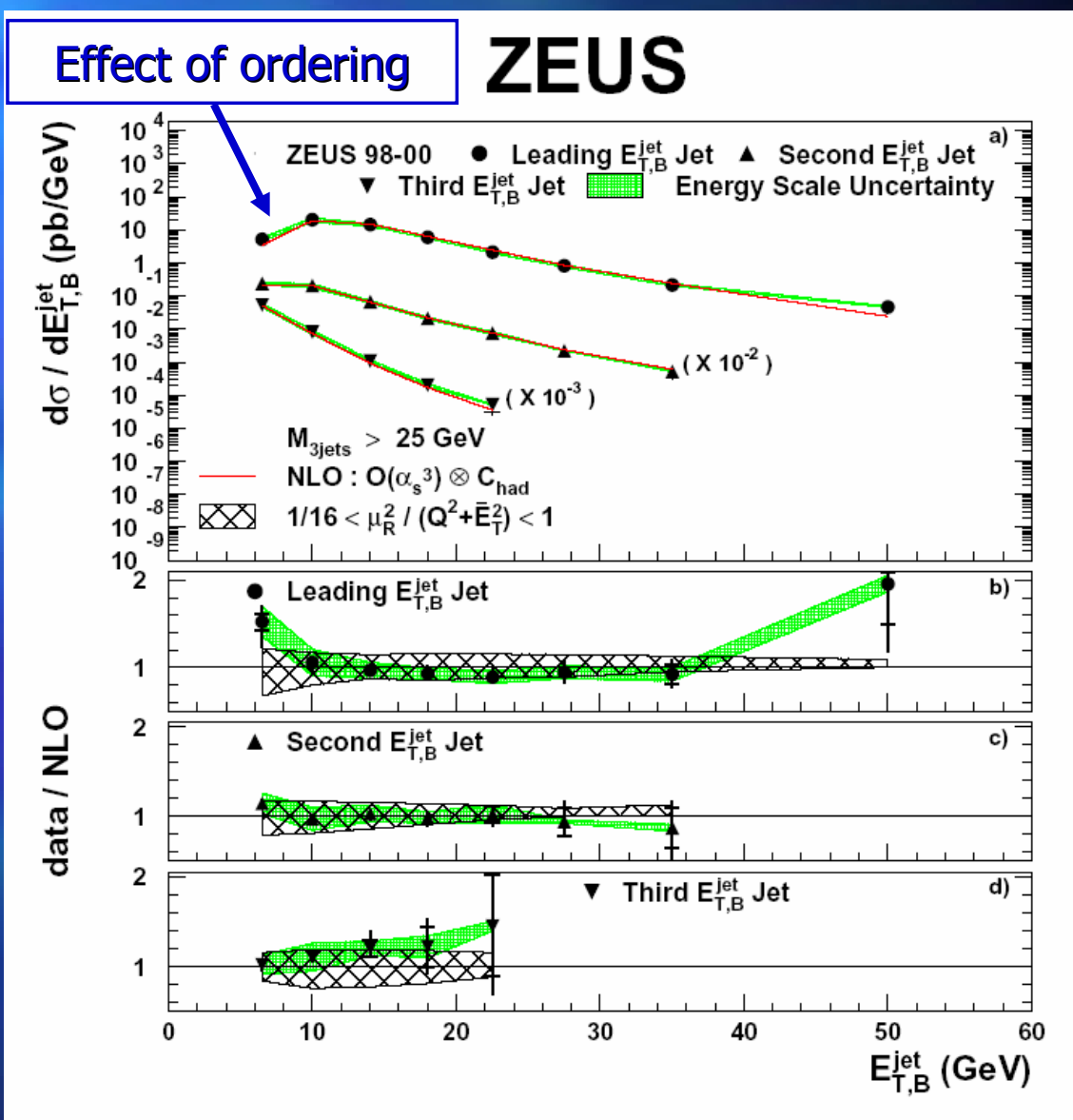




\section{Ratíjo of Trj-Jet to Di-Jet Productjon}

\lrcorner Correlated uncertainties largely cancel in ratjo

\lrcorner Ratio decreases with increasing $Q^{2}$

$\Rightarrow$ reflects decreasing strength of coupling

$\Rightarrow$ well described by theory

- Absolute ratio can be used to determine $\alpha_{s}\left(m_{z}\right)$

- systematics complementary to inclusive jet measurement

$\alpha_{s}=0.1179 \pm 0.0013$ (stat.) ${ }_{-0.0046}^{+0.0028}$ (exp.) ${ }_{-0.0046}^{+0.0064}$ (th.)

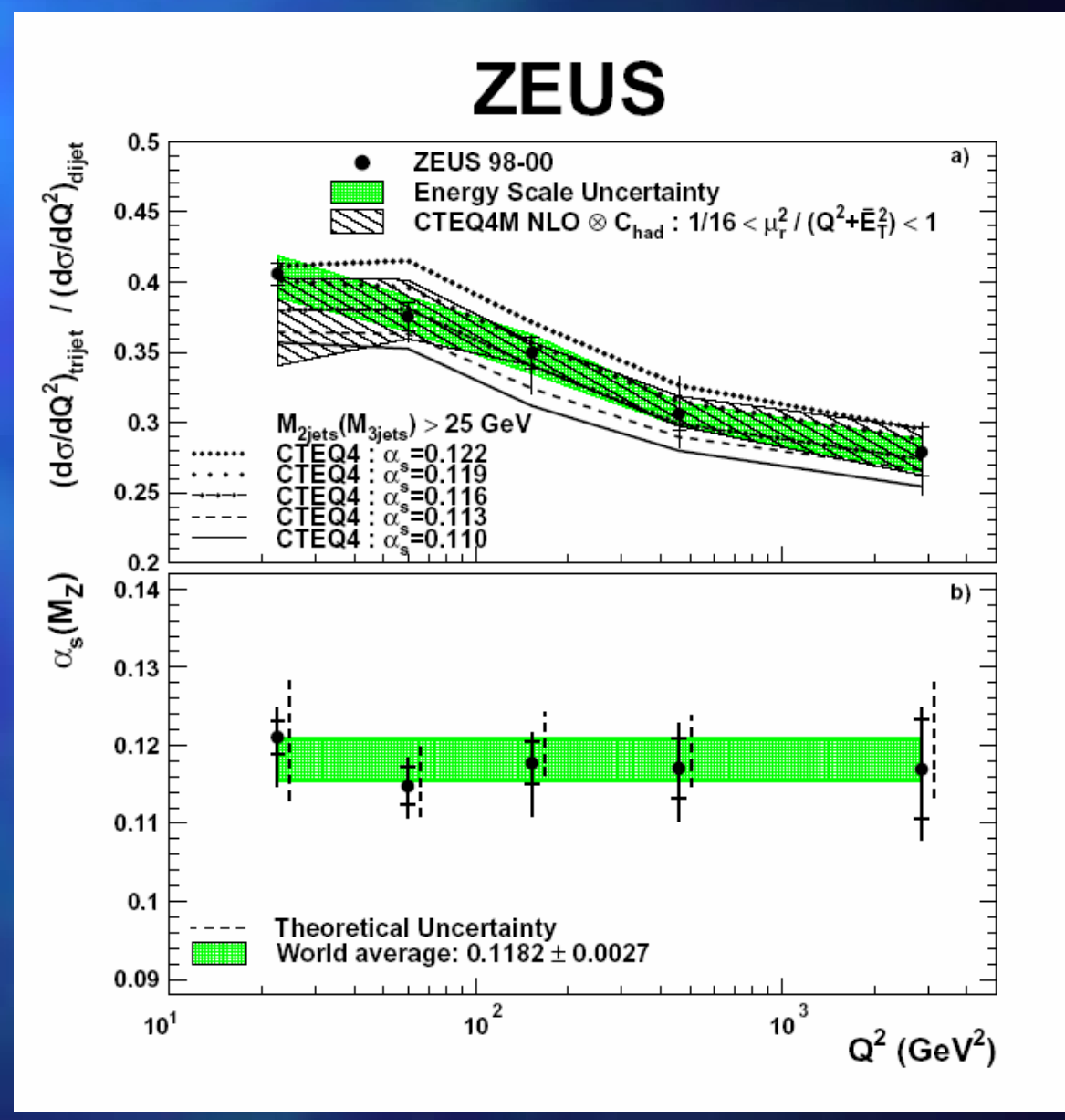




\section{$\mathrm{a}_{\mathrm{s}}$ Sussusnary}

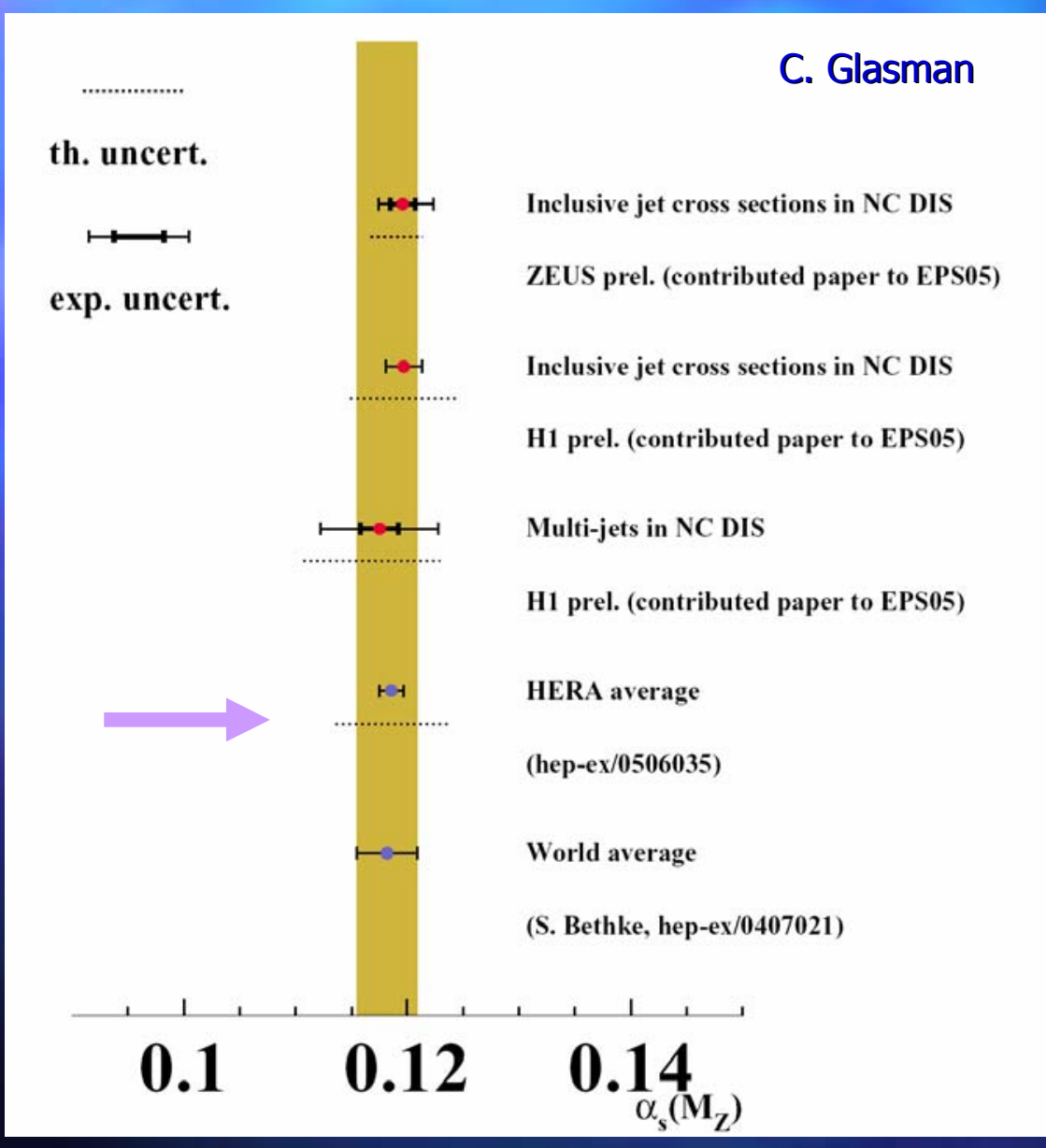

$-\alpha_{\mathrm{s}}$ measurements from HERA have reached an impressive level of precision

- need help from theory

$\square$ Consistent both internally \& with other experiments

- With more data to come from HERA-II $\rightarrow$ further improvement expected 

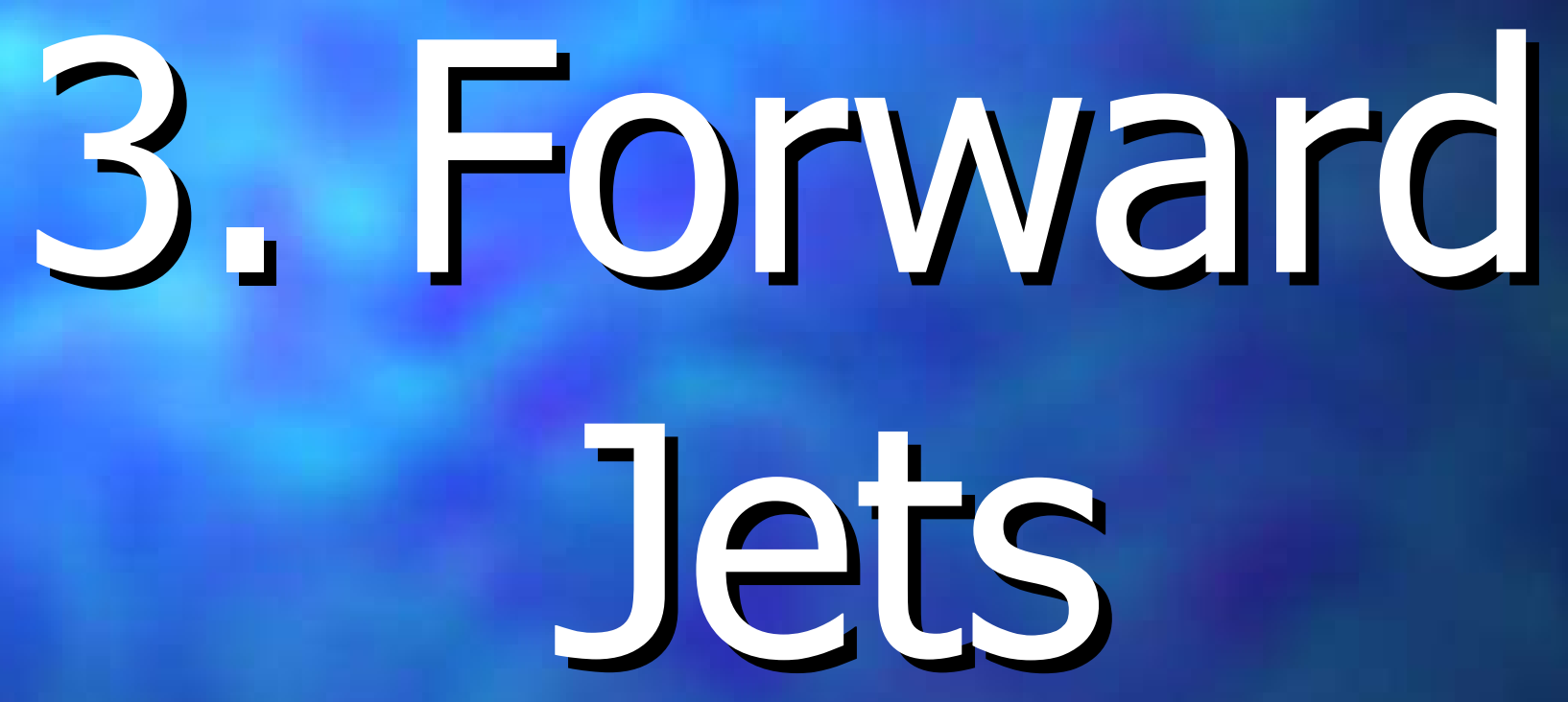


\section{Forward Jets}

\lrcorner Forward area is particularly sensicjue to details in evolution of parton cascade

\lrcorner At low $x$, we do not probe the valence structure of the proton, but rather see universal structure of QCD radiation at work

- signature: forward jet

- This enables us to examine different mechanisms of parton cascade evolutions

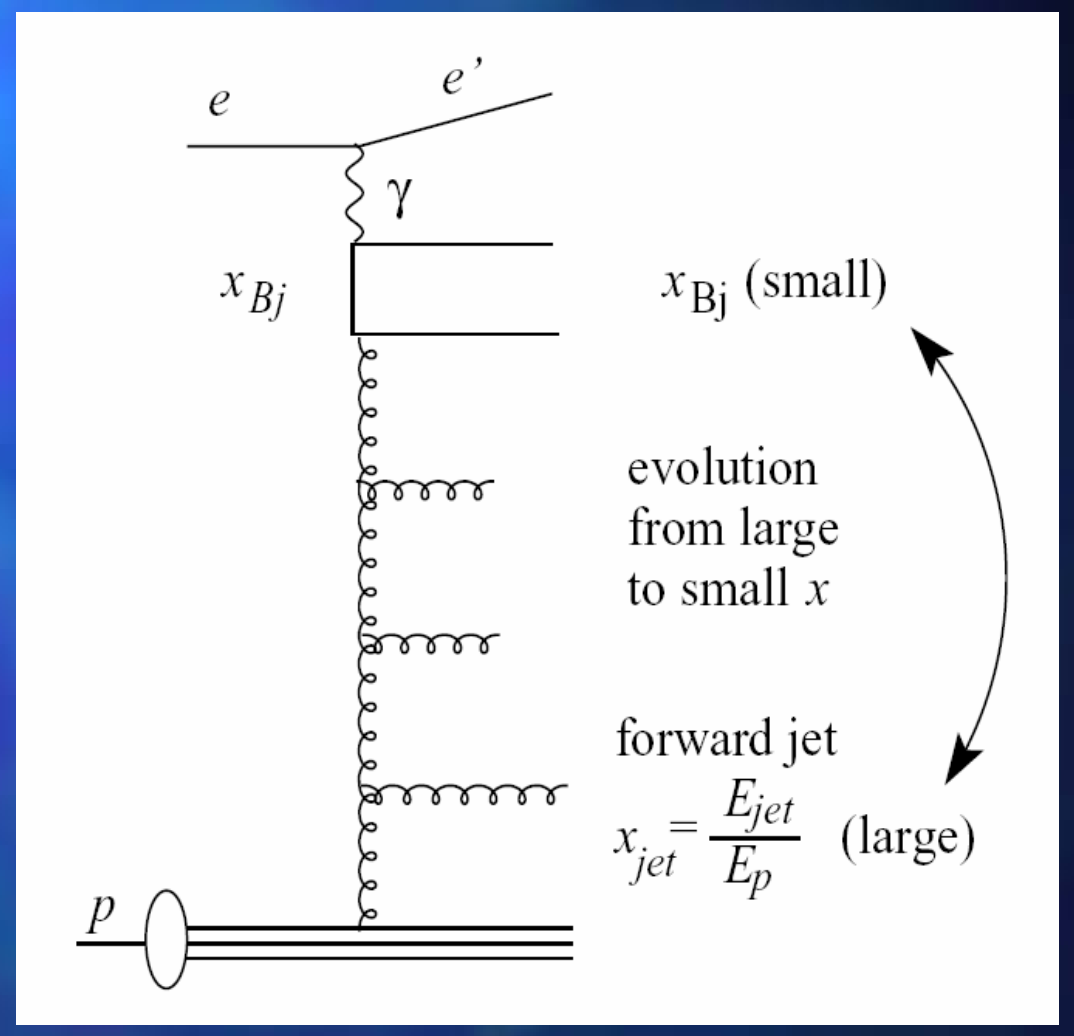




\section{Dynamsjics of Parton Evolution}
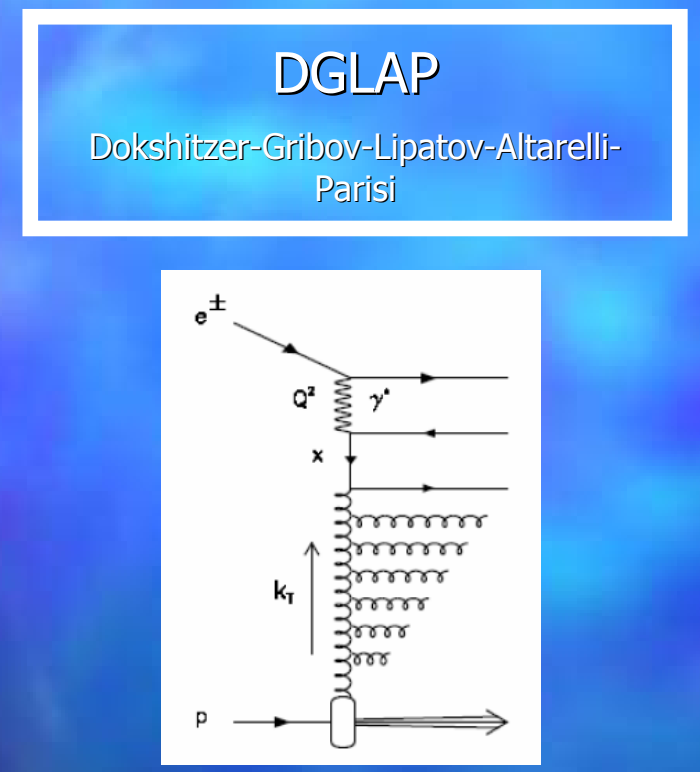

- Evolution in powers of $\ln \mathrm{Q}^{2}$

- Strongly orderered in $k_{T}$

- Well established at high $\mathrm{x}$ and $\mathrm{Q}^{2}$, but expected to break down at low $\mathrm{X}$

21-Sep-2006
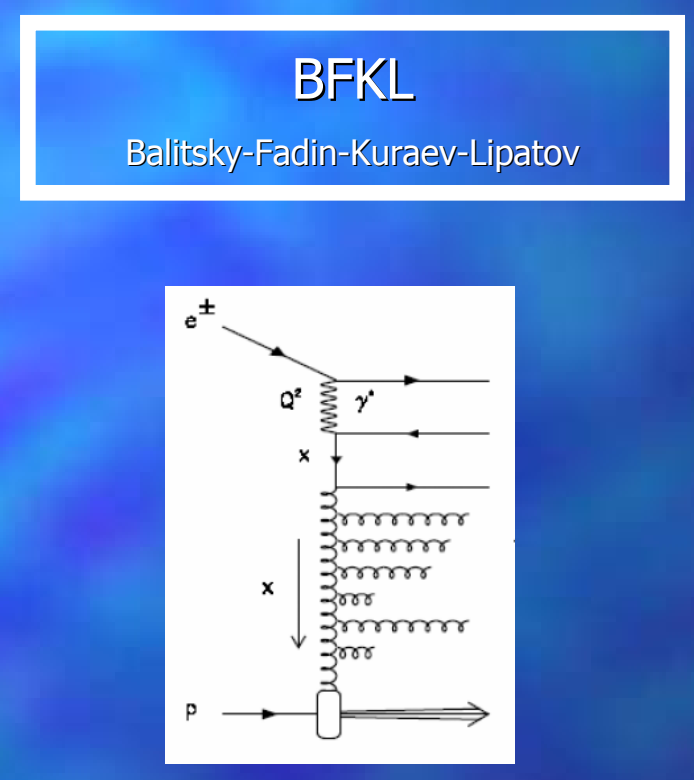

Evolution in powers of In $1 / x$

- Strongly orderered in $x$

- May be applicable at low $x$

\section{CCFM}

Ciafaloni-Catani-Fiorani-Marchesini

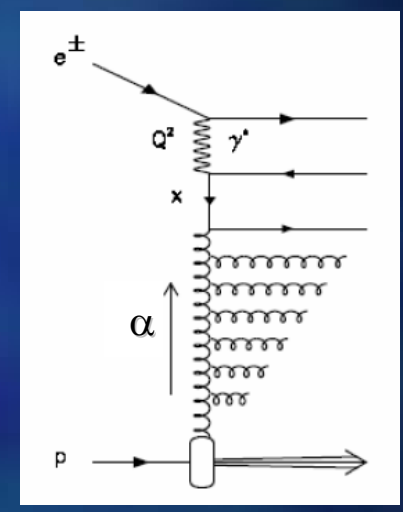

- Evolution in both $\ln \mathrm{Q}^{2}$ and In $1 / x$

- Bridge between DGLAP and BFKL

- Angular ordering

- May be applicable at low $\mathrm{x}$ 


\section{Forward Jet Measurements (DIS)}

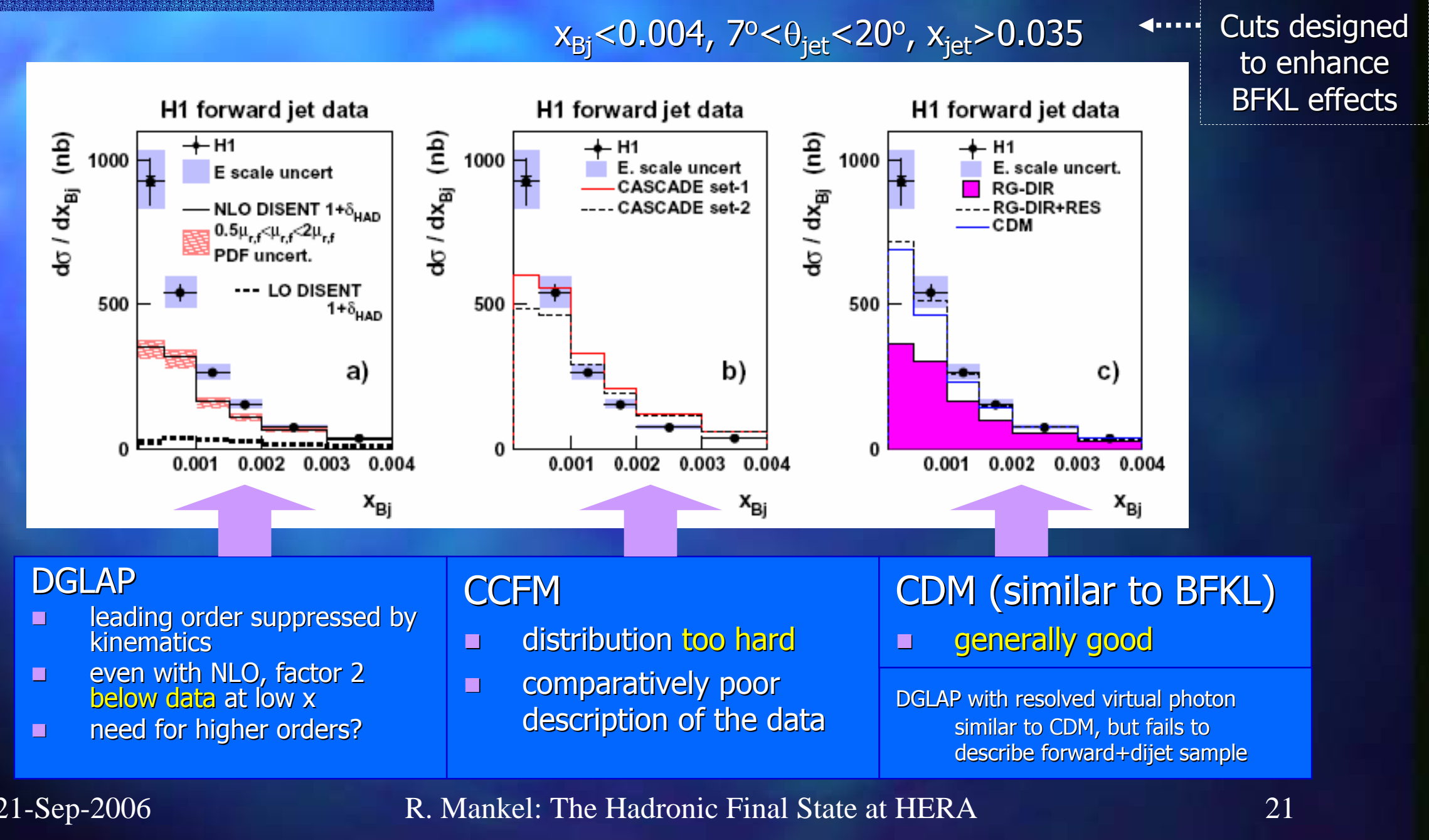




\section{Forward Jets Summary}

\lrcorner Ljmitations of the pure DGLAP approach clearly seen in the forward area

u higher order parton emissions break ordering scheme

- Calculations which include such processes (CDM) achieve better description of the data 
4. Charm \&

Jets in Photo-

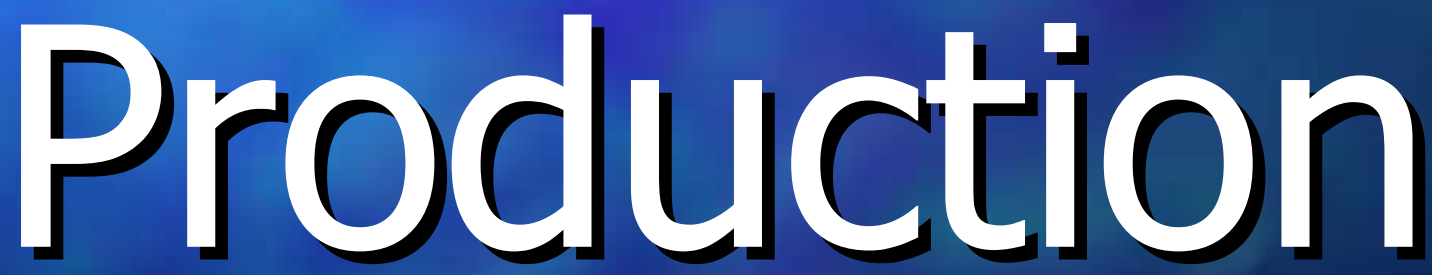




\section{D*-Jet Correlations in Photo- Production}

\lrcorner Charm quark mass provides hard scale even for quasi-real photon $\left(Q^{2} \sim 0\right)$

$\downarrow$ perturbative QCD (pQCD) applicable over full phase space

\lrcorner Several basic processes expected to contribute to photoproduction of charm

- Correlations between D* and a separate additional jet, or between two jets (one of them tagged by a $D^{*}$ ) allow a very fine-grained comparison of different theoretical approaches
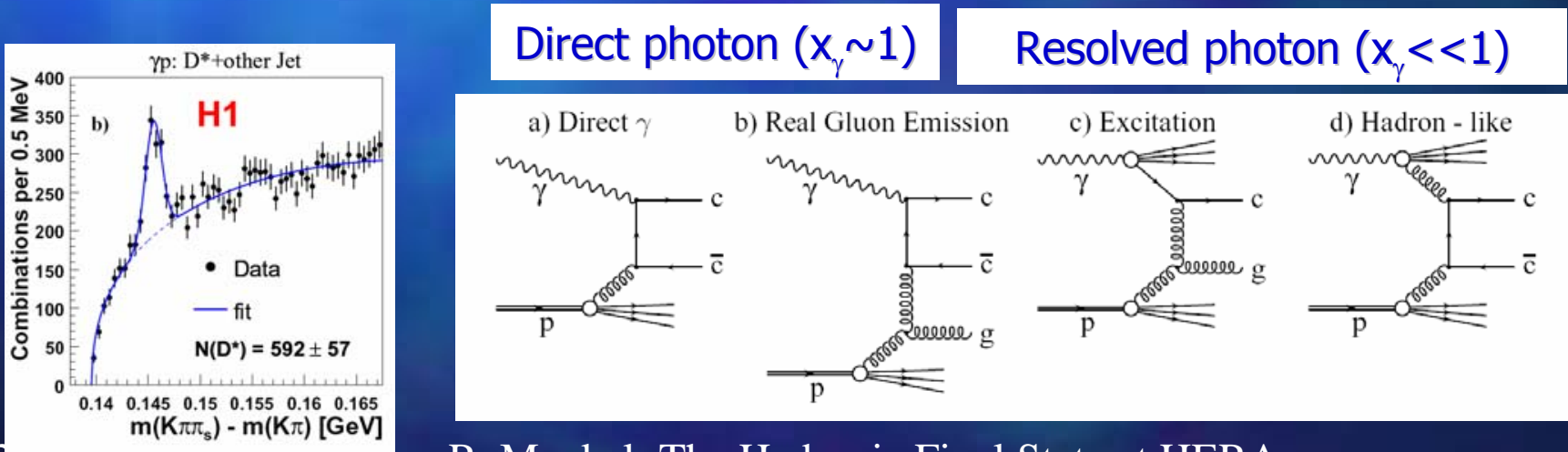


\section{How Models Treat Charm Productjon}

\lrcorner PYTHIIA: LO direct photon-gluon fusion, charm excitation \& hadjon-like, Higher order contributions simulated with leadinglog parton showers in collinear approach.

$\checkmark$ CASCADE: LO in $k_{T}$ factorization approach. Higher order corrections simulated with initial state parton showers (CCFM evolution)

- FMNR (Frixjone-Mangano-Nason-Ridolfi): NLO calculation $\left(\mathrm{O}\left(\alpha_{\mathrm{s}}^{2}\right)\right)$, massive scheme in collinear factorization approach

- ZMVFNS (Zero mass variable flavor number scheme) : NLO calculation $\left(\mathrm{O}\left(\alpha_{\mathrm{s}}^{2}\right)\right)$ in collinear approach, neglecting charm mass 


\section{D*-Jet Correlations: $\eta$ Spectra}

\lrcorner Data show marked diffierence in shape: jets on average more forward than $\mathrm{D}^{*}$

$\checkmark$ indicates presence of a hard non-charm parion in the forward direction

- dominant mechanism: hard gluon radiation from proton

- [a PYTHIA variant with only direct photon does not show this difference]

- All models include this \& describe effect well

$$
R=\frac{\frac{1}{\sigma_{v i s}^{\text {anc }} \frac{d \sigma^{\text {calc }}}{d Y}}}{\frac{1}{\sigma_{\text {data }}^{\text {data }}} \frac{d \sigma^{\text {data }}}{d Y}}
$$
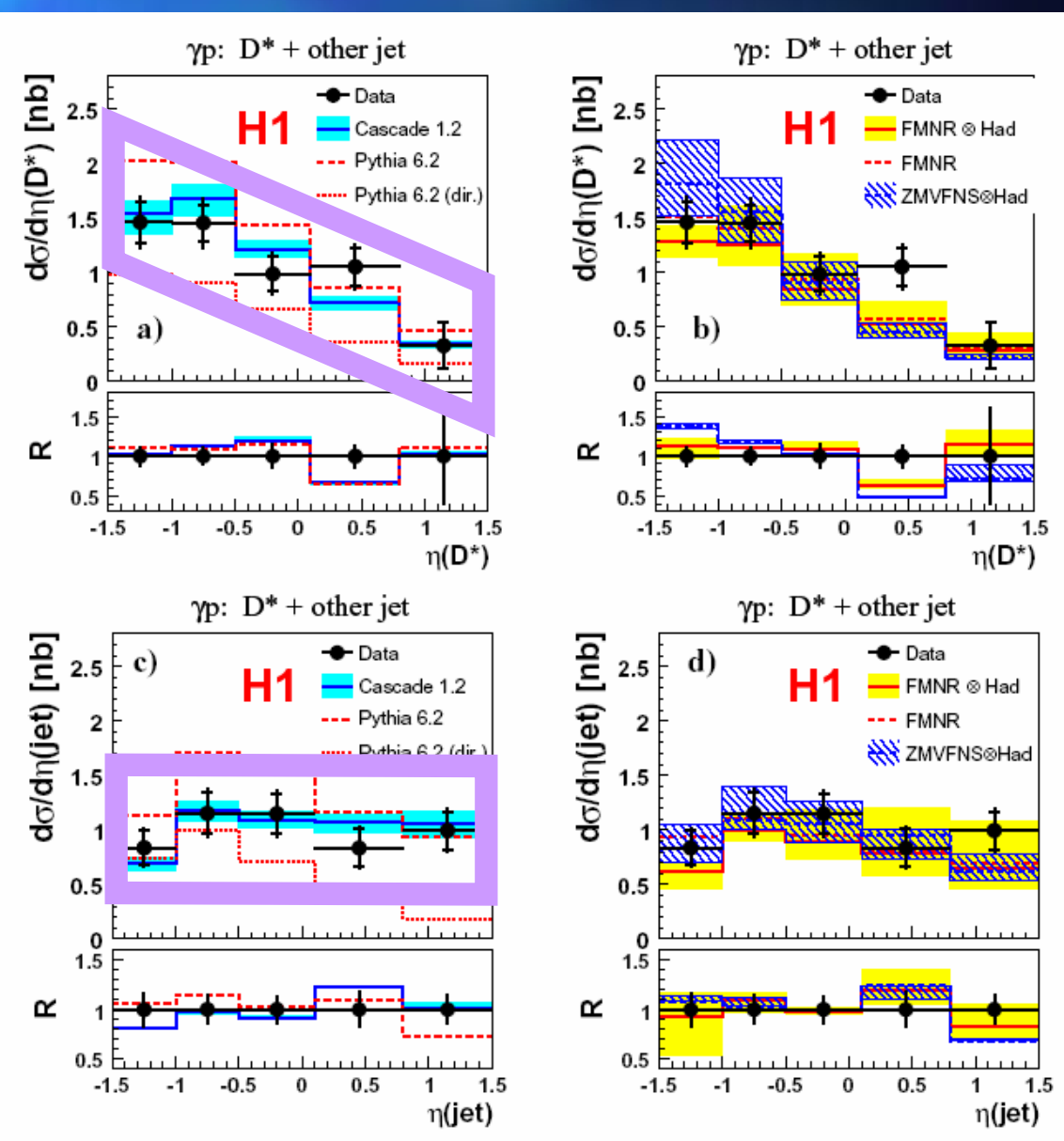

26

21-Sep-2006

R. Mankel: The Hadronic Final State at HERA 


\section{D*-Tagged Dijets: Transition from Resolved to Direct PHP}

\lrcorner $x_{y}^{\text {obs }}=$ firaction of photon energy partjcjpating in hard interaction

$\square$ x obs $\sim 1$ : dlirect PHP

$\square x_{\text {obs }}<<1$ : resolved PHP

$\downarrow$ Sensitive to gluon emission in initial state

- All calculations underestimate relative contribution in $x_{\gamma}^{\text {obs }}<0.6$ region

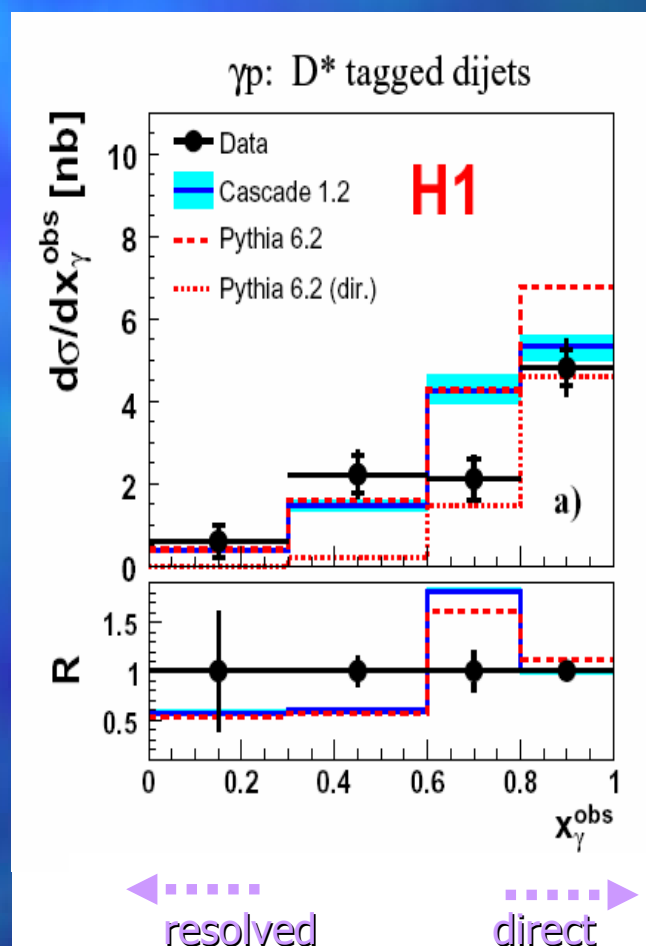

resolved

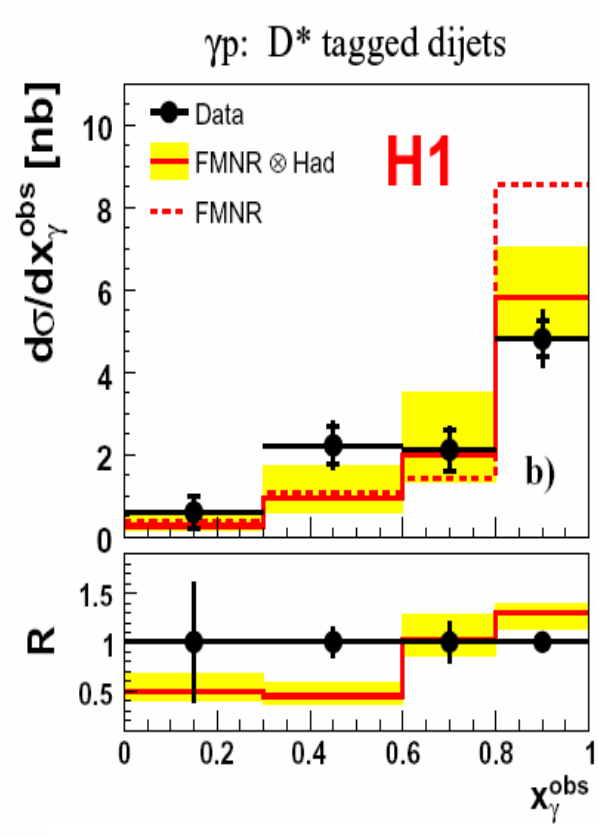

resolved 


\section{D*-Jet Correlations: Relative Azjnutís Angle}

\lrcorner$\quad$ In collinear approximation, process $y g \rightarrow c c$ should lead to back-to-back topology

\lrcorner$\quad$ But data show: only $25 \%$ of cross section are strictly backto-back

$\checkmark \quad$ Remainder can only be described with significant contributions from higher order QCD racliation

$\Rightarrow \quad$ Neither PYTHIA nor CASCADE describe full range

$\Rightarrow$ NLO calculation too low for $\Delta \varphi<120^{\circ} \rightarrow$ relevance of higher order contributions

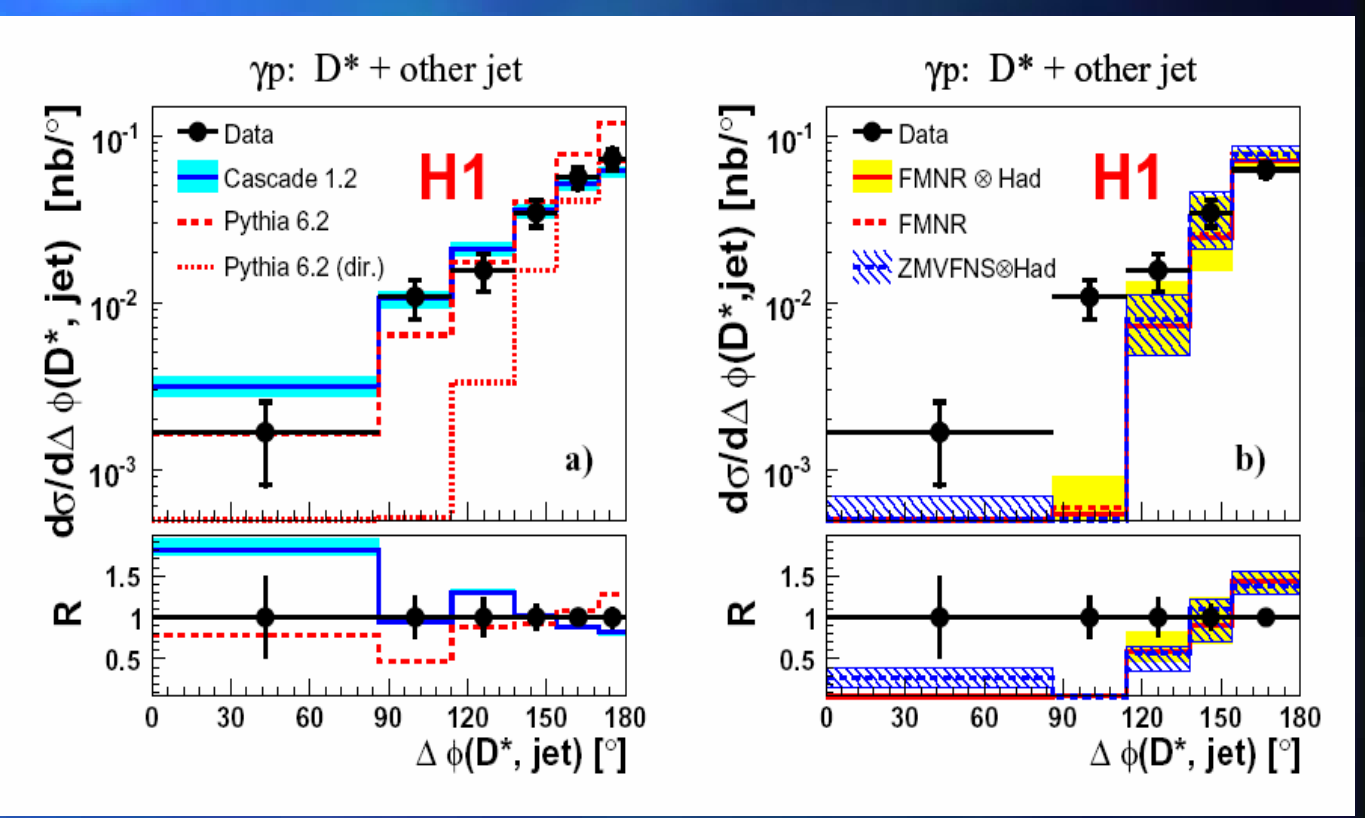

$\Rightarrow$ Rich testing ground for QCD, challenging for theory 

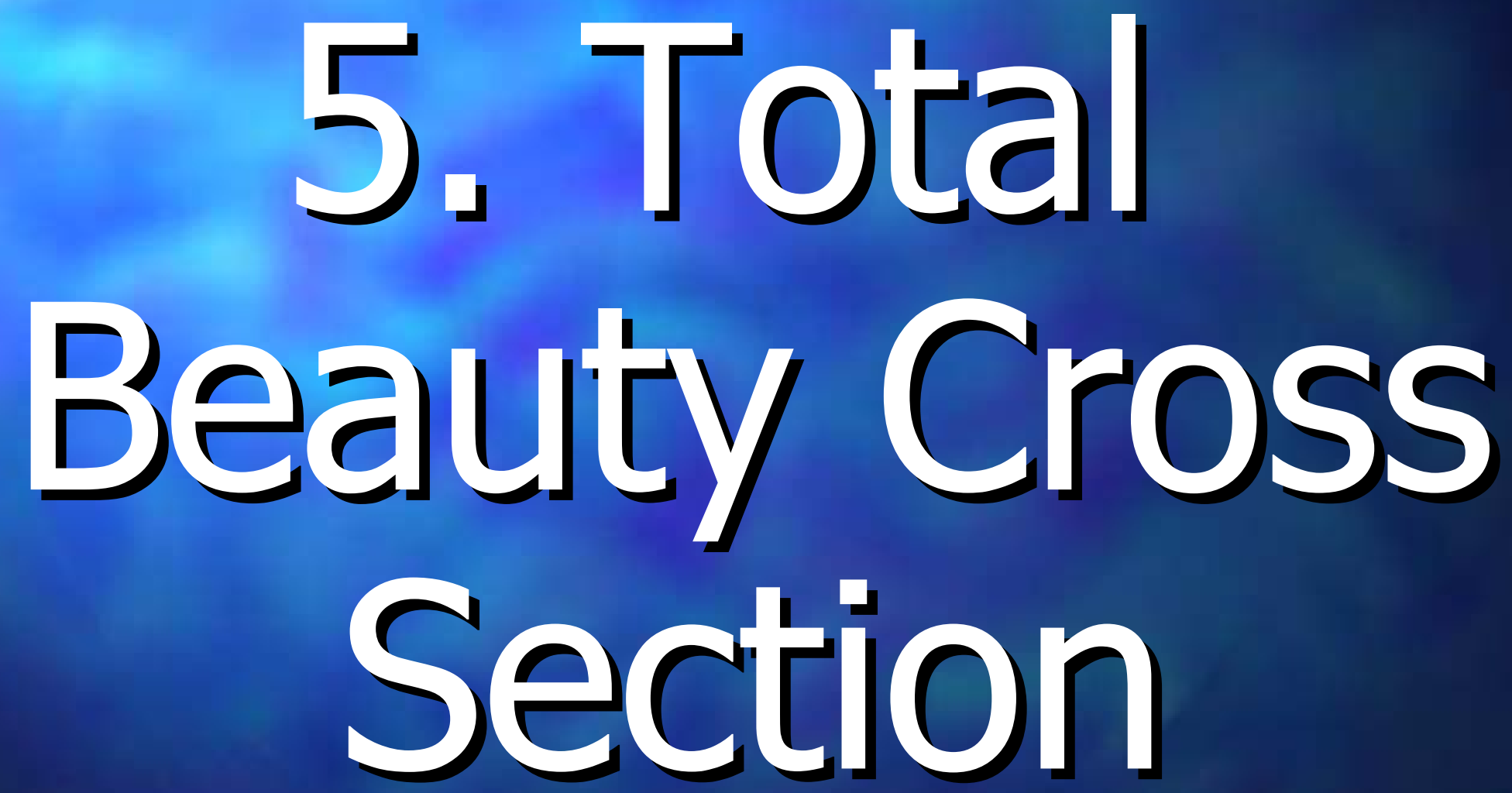


\section{Total Beauty Cross Section}

\lrcorner Very stringent QCD test

\lrcorner large $m_{b} \rightarrow$ pQCD reliable in full phase space?

\lrcorner Measurements in pp, $\gamma y$, $\pi \mathrm{N}$ and $\mathrm{pN}$ have shown large discrepancjes

$\sqcup$ Experimental challlenge:

- beauty often tagged with high $\mathrm{p}_{\mathrm{T}}$ electron or muon (secondary vertex, or $p_{\mathrm{T}}$ relative to jet)

- measurement restricted to high $\mathrm{p}_{\mathrm{T}} \mathrm{b}$ quark $\rightarrow$ extrapolation uncertainty

- Alternative: correlation signature

- example: di-muon

- Study of di-muon event signatures allows to use low $p_{t}^{\mu}$ thresholds

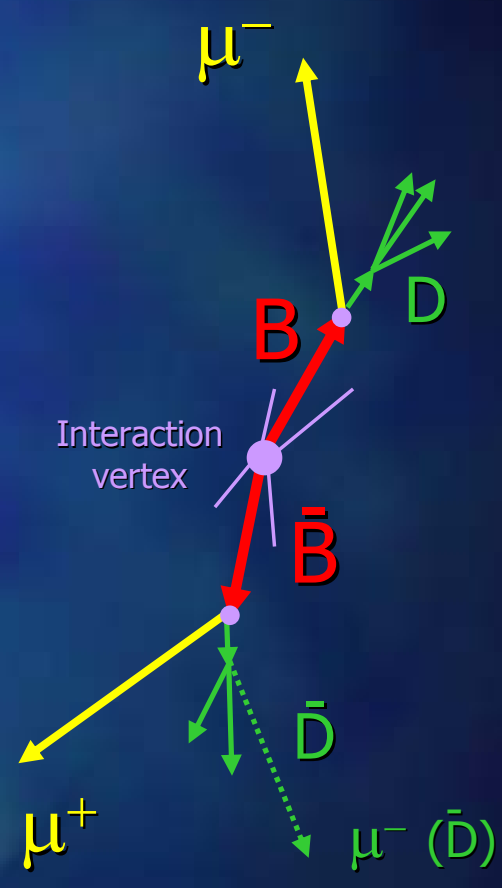

$\rightarrow$ measure the total bb cross section 


\section{Extraction of Beauty Signal}

\lrcorner Light flavor background sinnilas in $(+-)$ and $( \pm \pm)$ mass spectra $\rightarrow$ exploit for subtraction

\lrcorner Bethe-Hejtler and quarkonia background suppressed by non-isolation requirement

$\checkmark$ Bethe-Heitler, quarkonia and cc background subtracted using MC (PYTHIA, RAPGAP, HERWIG, GRAPE)

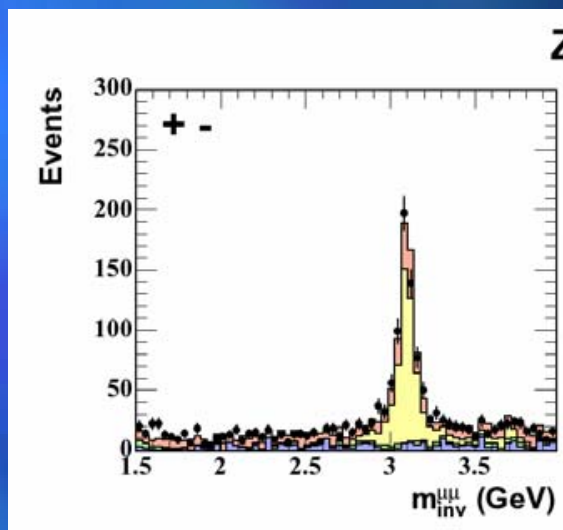

ZEUS
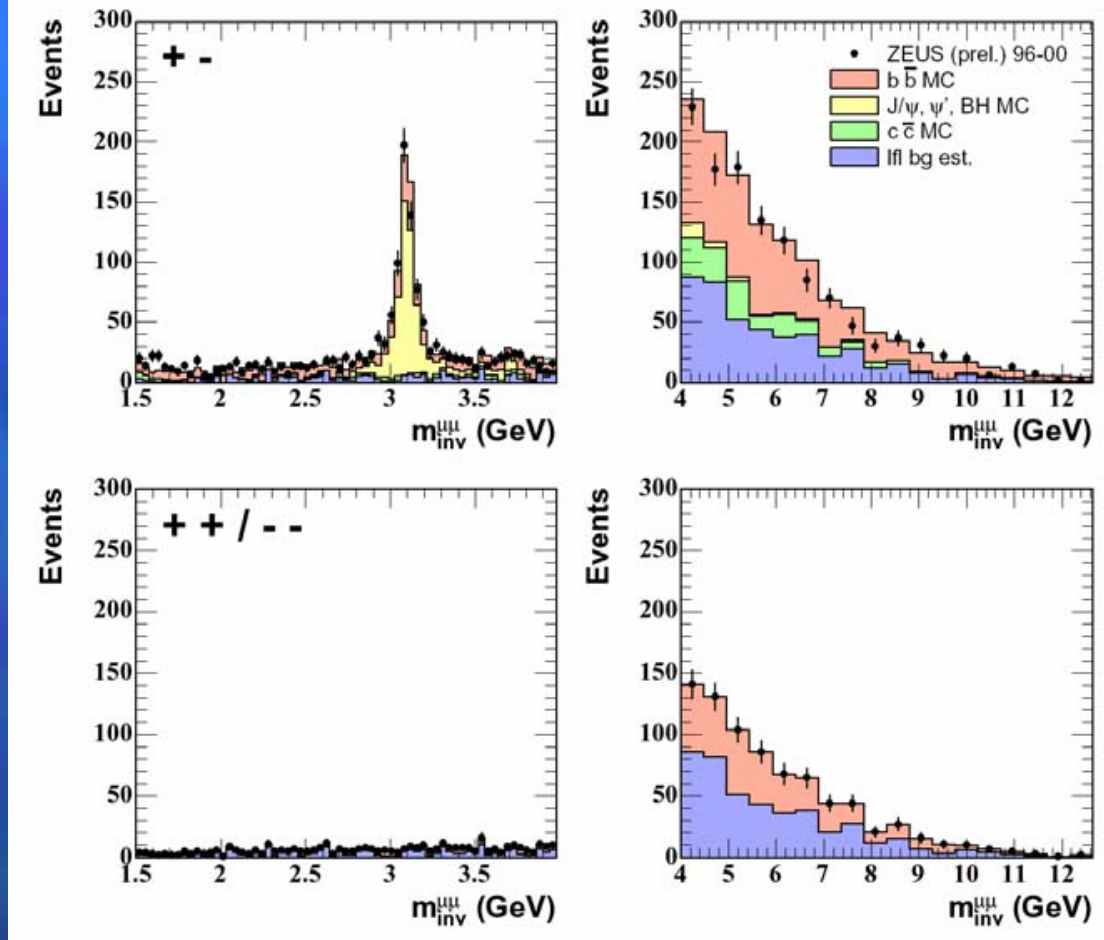


\section{Bealuty from Di-Muons: Accessible Quark $p_{\mathrm{T}}$ Range}

$\mathrm{p}_{\mathrm{T}}$ djistribution of tagged b quarks

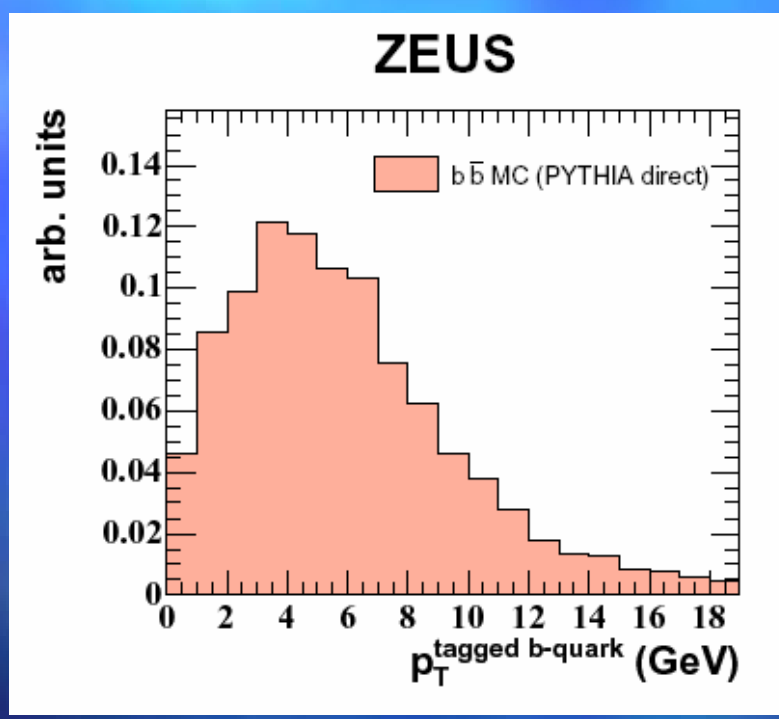

$\Rightarrow$ Method is sensitive down to $\mathrm{P}_{\mathrm{T}}(\mathrm{b}) \sim 0$

$\Rightarrow$ Small extrapolation uncertainty 


\section{b' Cross Section from Di-Muon Events}

$\sigma_{\text {tot }}(e p \rightarrow b \bar{b} X)(\sqrt{s}=318 \mathrm{GeV})=16.1 \pm 1.8(\text { stat })_{-4.8}^{+5.3}$ (syst) $n b$

NLO QCD prediction: $6.8_{-1.7}^{+3.0} \mathrm{nb}$ PHP: $5.8 \mathrm{nb} \quad$ (FMNR,CTEQ5M)
Note:

PYTHIA+RAPGAP scaled by $1.95 x$

For muons from $b$ decays

$\Rightarrow$ Wide phase space

$\Rightarrow$ Good agreement in shape

$\Rightarrow$ Normalization underestimated by theory
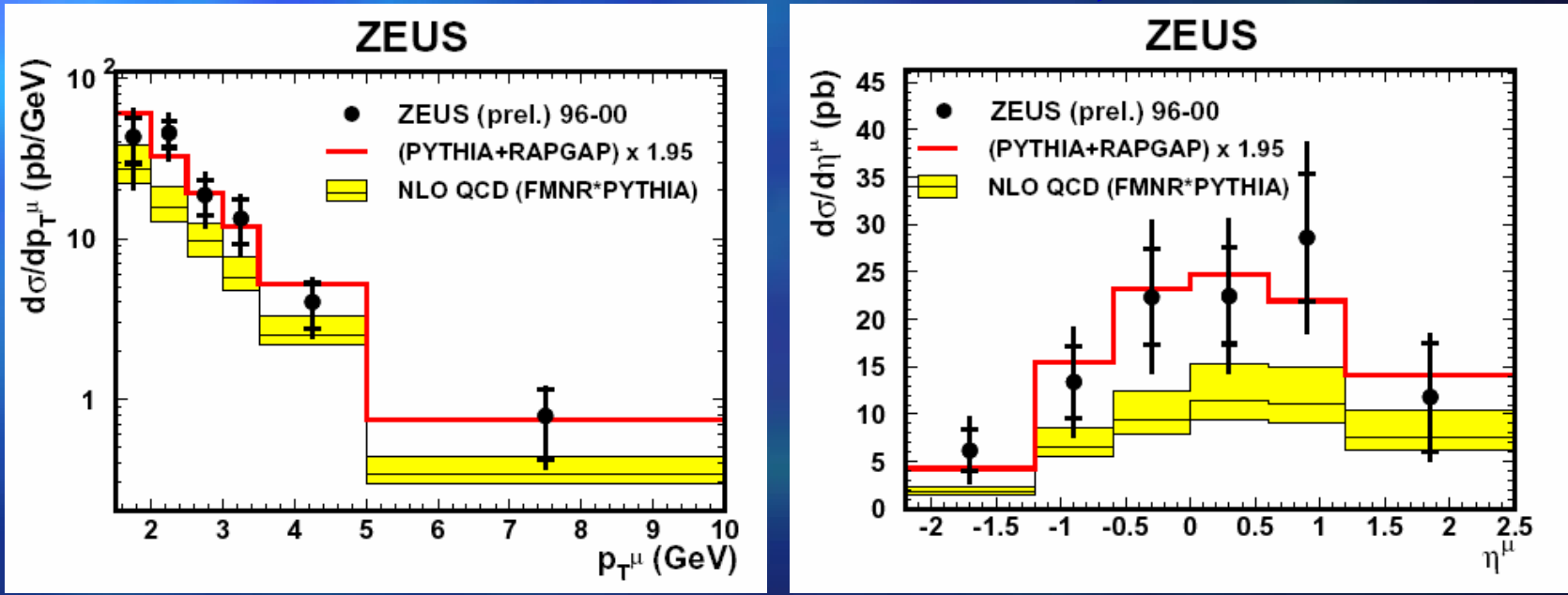


\section{HERA bi Cross Section vs Theory}

$\Rightarrow$ Wide range of measulirements available

$\Rightarrow$ Measurenents tend to be larger than NLO

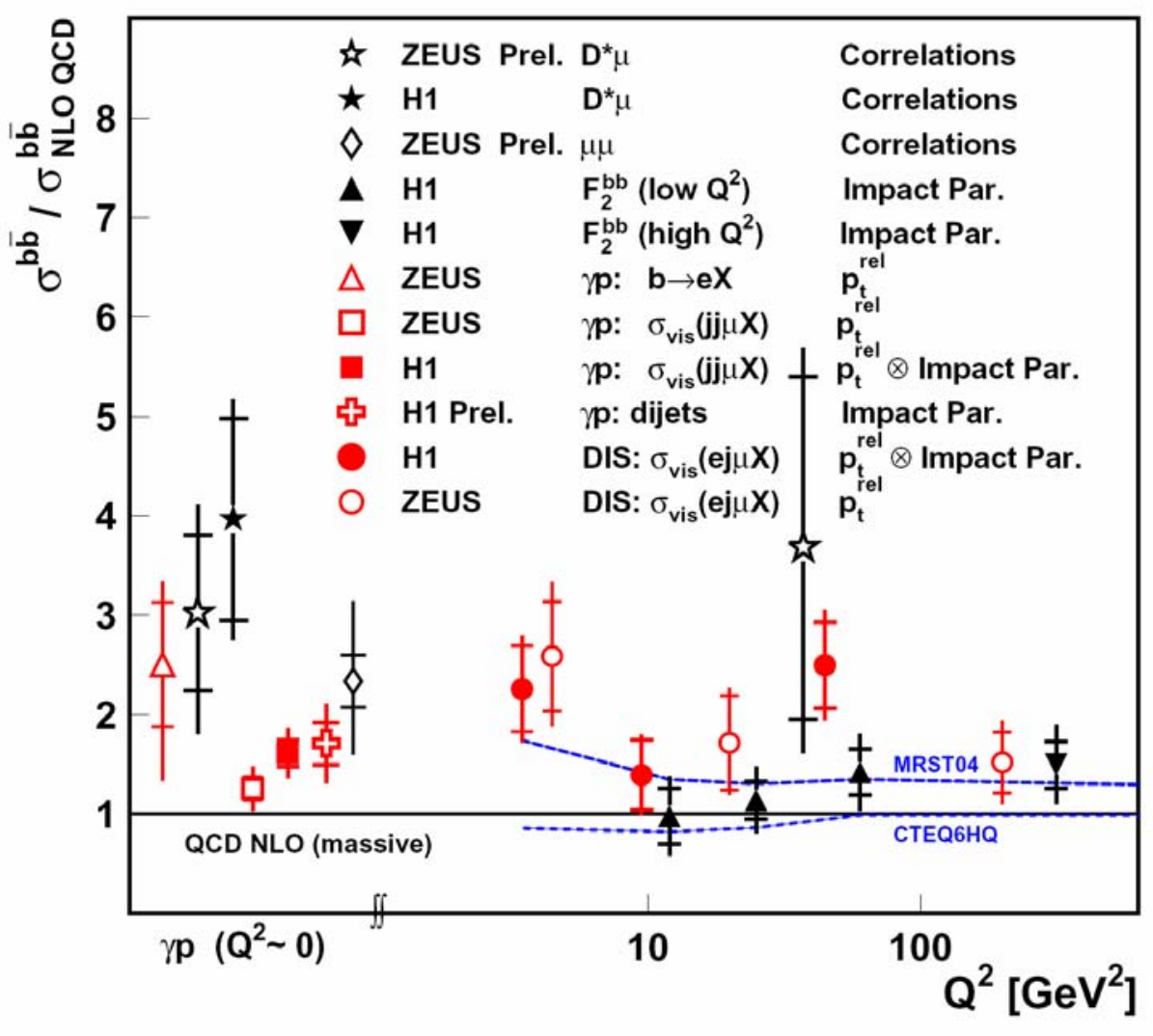



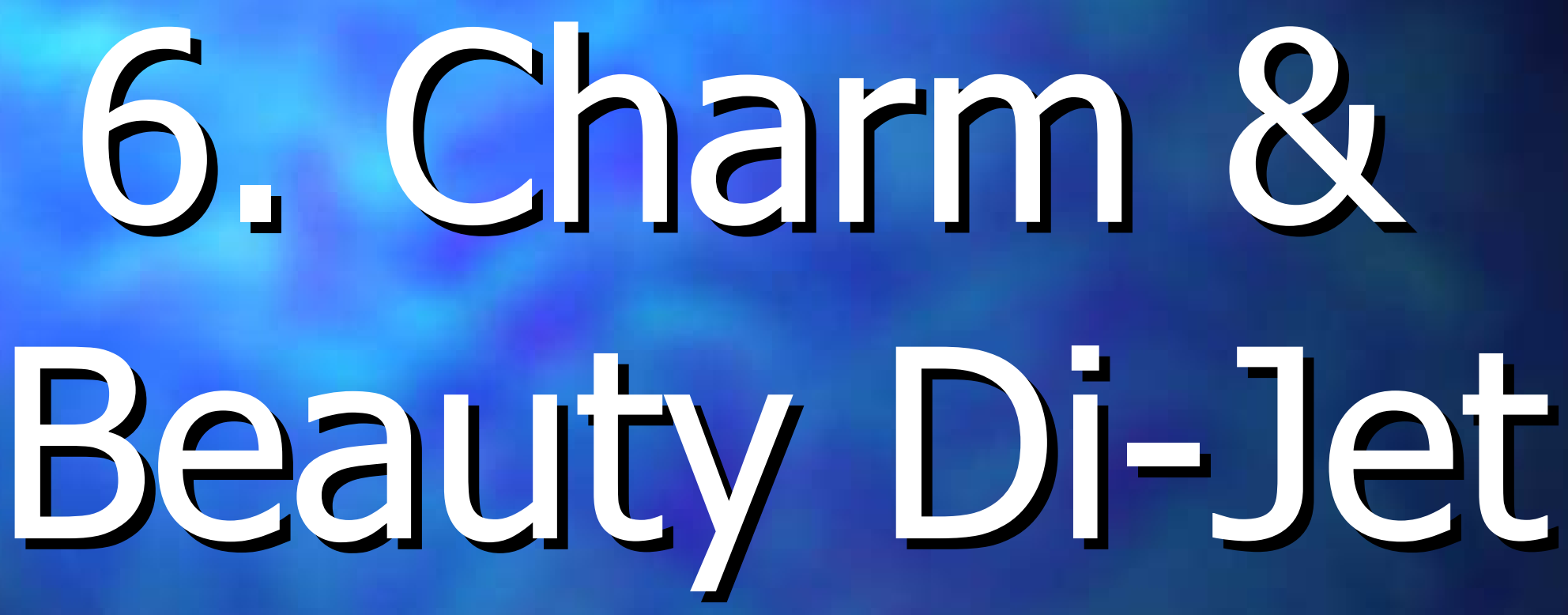


\section{Charm \& Beauty Di-Jet Cross Sectjons in PHP}

\lrcorner Typical topology for charm \& beauty production: $\geq 2$ jets

\lrcorner A very elegaint way to identify heavy quark production is to use lifetime tags

$\checkmark c+b$ lifietime leads to significantly positive values of impact parameter $\delta$ of charged tracks

- can be measured with high resolution sillicon vertex detectors

- signed according to jet direction

- Allows simultaneous determination of charm $\&$ beauty rates in PHP

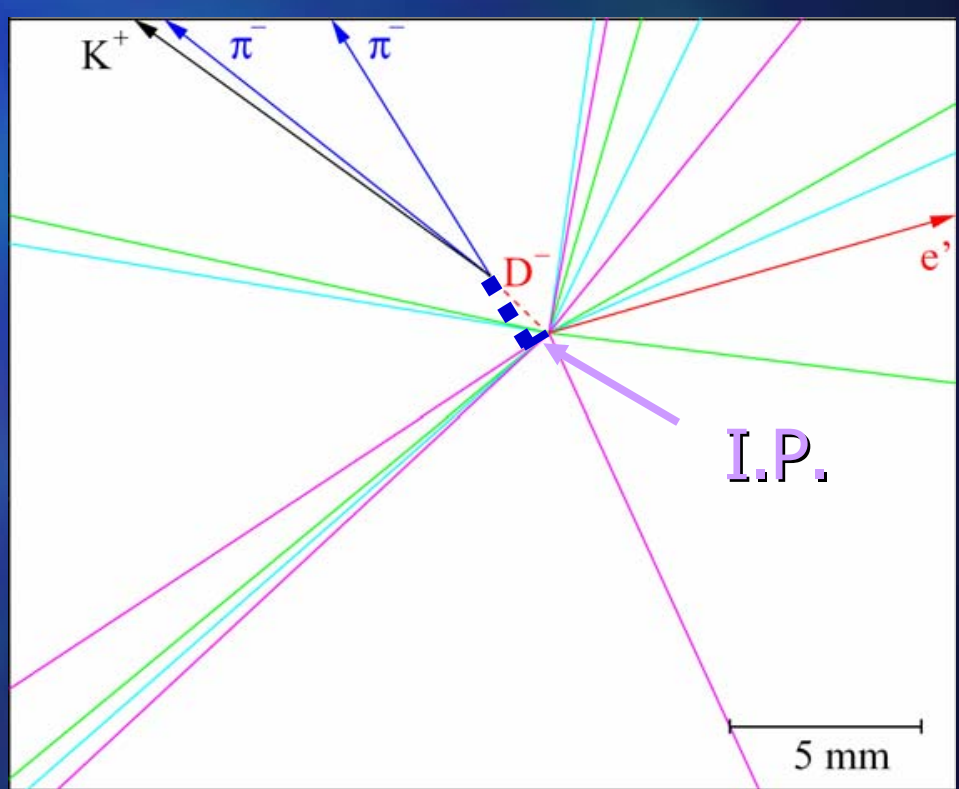




\section{Chajrn \& Beauty in PHP Di-Jet $\left(\cos t^{\prime} d\right)$}
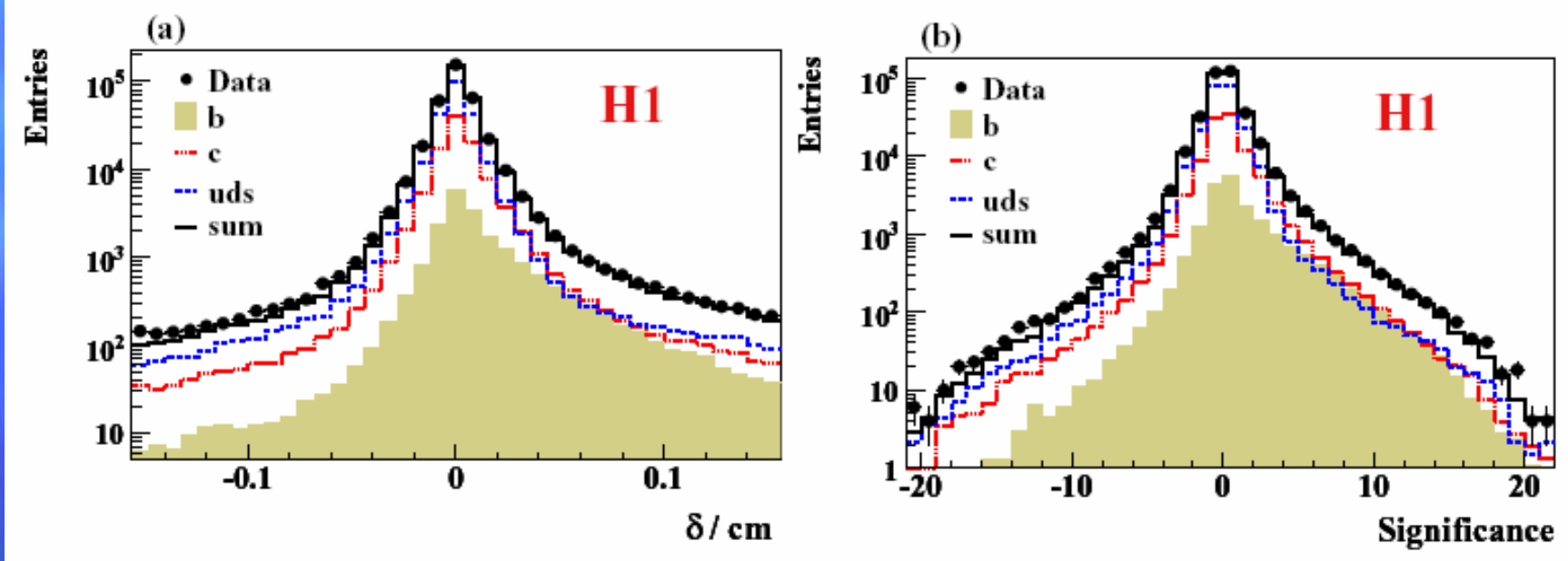

Tracks with $\mathrm{p}_{\mathrm{T}}>0.5 \mathrm{GeV}$, $30^{\circ}<\theta<150^{\circ}$, \#CST hits $(r \varphi) \geq 2$

Significance $=\frac{\delta}{\sigma(\delta)}$

$\Rightarrow$ Clear excess at positive impact parameter (significance)

- Since $m_{b}>>m_{c \text {, }}$ decays of beauty hadrons have significantly higher number of tracks on average

- Divide into 2 samples according to tracks associated to jet:

- \#tracks=1: charm enriched

- \#tracks $>1$ : beauty enriched 


\section{Charm \& Beauty in PHP Di-Jet:}

\section{Cross Sectjons}

\lrcorner Cross section:

\lrcorner$\quad$ NLO (FMNR) agrees for charm, but factor 1.8 too low for beauty

- PYTHIIA, CASCADE similar

\lrcorner Shapes of p jet and njet (not shown) reasonably well described

$-\quad x^{o b s}=$ fraction of photon energy particjpating in hard interaction

- At low $x^{\text {obs }}$ (resolved photon regime), NLO calculation strongly underestimates the beauty cross section

- PYTHIA agrees in shape

- At $x_{\text {obs }}>0.85$ (direct photon regime), models work generally well ( $\leftrightarrow$ photon gluon fusion)

$\mathrm{Q}^{2}<1 \mathrm{GeV}^{2}, 0.15<\mathrm{y}<0.8$, $\mathrm{p}_{\top}^{\text {jet1(2) }}>11(8) \mathrm{GeV}_{\boldsymbol{J}}-0.9<\eta^{\text {jet1}(2)}<1.3$

\begin{tabular}{|r||c|c|}
\hline & Charm [pb] & Beauty $[\mathrm{pb}]$ \\
\hline Data & $702 \pm 67($ stat. $) \pm 95($ syst. $)$ & $150 \pm 17($ stat. $) \pm 33($ syst. $)$ \\
FMNR & $500_{-99}^{+173}$ & $83_{-14}^{+19}$ \\
PYTHIA & 484 & 76 \\
CASCADE & 438 & 80 \\
\hline
\end{tabular}
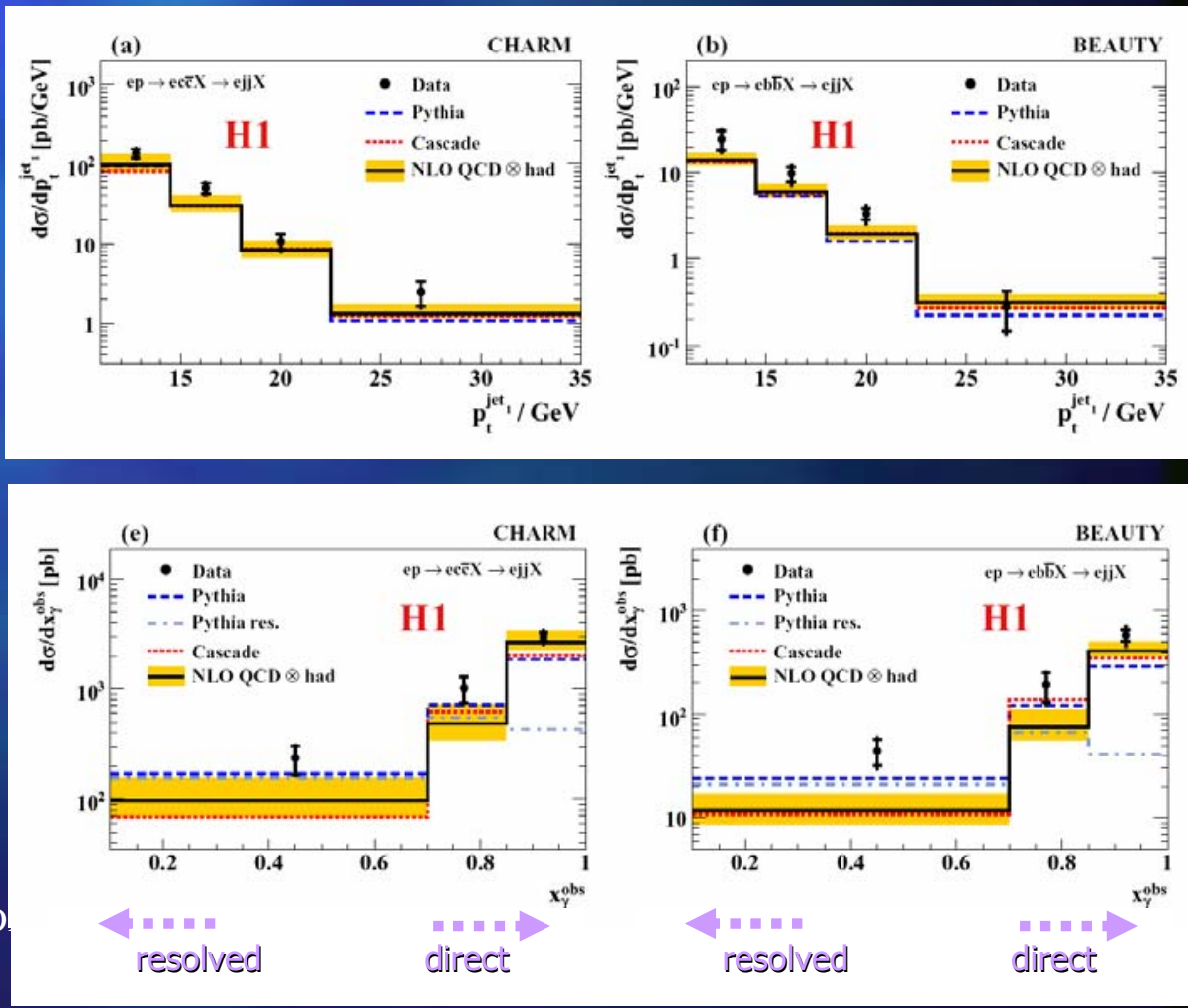


\section{Sunnjsajry}

\lrcorner Wealth of measurements from HERA on structure of hasdronjic final staite

$\downarrow$ only a small selection presented

- unique facillity for QCD studies

\lrcorner NLO largely successful in describing experimental data

$\checkmark$ Some challenging frontiers identified

- QCD dynamics in vicinity of proton remnant (low $x$ regime)

- resolved photo-production

- beauty cross section

- With large HERA-II data sample, and improvements in theory, expect further insights in QCD frontiers regarding the hadronic final state 